\title{
COINDUCTION FUNCTOR IN REPRESENTATION STABILITY THEORY
}

\author{
WEE LIANG GAN AND LIPING LI
}

\begin{abstract}
We study the coinduction functor on the category of FI-modules and its variants. Using the coinduction functor, we give new proofs of (generalizations of) various results on homological properties of FI-modules. We also prove that any finitely generated projective VI-module over a field of characteristic 0 is injective.
\end{abstract}

\section{Contents}

1. Introduction

2. Preliminaries

3. Injectivity of $k \mathrm{C} e_{0}$

4. Restriction and coinduction along genetic functors

5. Structure of $\mathrm{Q}\left(k \mathcal{C} e_{m}\right)$ when $\mathcal{C}$ is $\mathrm{FI}_{G}$

6. Structure of $\mathrm{Q}\left(k \mathrm{C} e_{m}\right)$ when $\mathrm{C}$ is VI

7. Applications of coinduction functor

8. Homological approach to representation stability

References

\section{INTRODUCTION}

1.1. Conventions. Let $k$ be a commutative ring. By a category, we shall always mean a small category. If $\mathcal{C}$ is a category, a $\mathcal{C}$-module over $k$ is a functor from $\mathcal{C}$ to the category of $k$-modules. We shall refer to $\mathcal{C}$-modules over $k$ simply as $\mathcal{C}$-modules. A morphism of $\mathcal{C}$-modules is a natural transformation of functors. We shall write $\mathcal{C}$-Mod for the category of $\mathcal{C}$-modules, and $\mathcal{C}$-mod for the category of finitely generated $\mathcal{C}$-modules.

If $\mathcal{C}$ is a category and $m, n \in \mathrm{Ob}(\mathcal{C})$, we shall write $k \mathrm{C}(m, n)$ for the free $k$-module on the set $\mathcal{C}(m, n)$. Let $k \mathfrak{C}=\bigoplus_{m, n \in \mathrm{Ob}(\mathcal{C})} k \mathcal{C}(m, n)$ and denote by $e_{n} \in \mathcal{C}(n, n)$ the identity morphism of $n \in \mathrm{Ob}(\mathcal{C})$. We have a natural algebra structure on $k \mathfrak{C}$ where the product of $\alpha \in \mathfrak{C}(r, n)$ and $\beta \in \mathfrak{C}(m, l)$ is defined to be the composition $\alpha \beta$ if $r=l$; it is defined to be 0 if $r \neq l$. A $k \mathcal{C}$-module $V$ is said to be graded if $V=\bigoplus_{n \in \mathrm{Ob}(\mathrm{C})} e_{n} V$. If $V$ is a $\mathcal{C}$-module, then $\bigoplus_{n \in \mathrm{Ob}(C)} V(n)$ has a natural structure of a graded $k \mathrm{C}$-module which we shall also denote by $V$. Conversely, any graded $k \mathcal{C}$-module $V$ defines a natural $\mathcal{C}$-module denoted again by $V$, with $V(n)=e_{n} V$. Thus, we shall not distinguish between the notion of $\mathcal{C}$-modules and the notion of graded $k \mathrm{C}$-modules. 
1.2. Main results. Let $\mathbb{Z}_{+}$be the set of non-negative integers. For any $n \in \mathbb{Z}_{+}$, we write $[n]$ for the set $\{1, \ldots, n\}$; in particular, $[0]=\emptyset$. Let $G$ be a finite group.

Definition 1.1. Let $\mathrm{FI}_{G}$ be the category whose set of objects is $\mathbb{Z}_{+}$, and whose morphisms from $m$ to $n$ are all pairs $(f, c)$ where $f:[m] \rightarrow[n]$ is an injective map and $c:[m] \rightarrow G$ is an arbitrary map. The composition of morphisms $\left(f_{1}, c_{1}\right) \in \mathrm{FI}_{G}(m, l)$ and $\left(f_{2}, c_{2}\right) \in \mathrm{FI}_{G}(l, n)$ is defined by $\left(f_{2}, c_{2}\right)\left(f_{1}, c_{1}\right)=\left(f_{3}, c_{3}\right)$ where

$$
f_{3}(t)=f_{2}\left(f_{1}(t)\right), \quad c_{3}(t)=c_{2}\left(f_{1}(t)\right) c_{1}(t), \quad \text { for all } t \in[m] .
$$

We write $\mathrm{FI}$ for $\mathrm{FI}_{G}$ when $G$ is trivial.

Let $\mathbb{F}$ be a finite field.

Definition 1.2. Let VI be the category whose set of objects is $\mathbb{Z}_{+}$, and whose morphisms from $m$ to $n$ are all injective linear maps from $\mathbb{F}^{m}$ to $\mathbb{F}^{n}$. The composition of morphisms is defined to be the composition of maps.

Suppose $\mathcal{C}$ is the category $\mathrm{FI}_{G}$ or VI. There is a natural monoidal structure $\odot$ on $\mathcal{C}$ defined on objects by $m \odot n=m+n$. Define a functor $\iota: \mathcal{C} \rightarrow \mathcal{C}$ by

$$
\iota(n)=1 \odot n, \quad \iota(\alpha)=e_{1} \odot \alpha, \quad \text { for each } n \in \operatorname{Ob}(\mathcal{C}), \alpha \in \operatorname{Mor}(\mathcal{C}) .
$$

The functor $\iota$ is faithful and gives rise to a restriction functor:

$$
\mathrm{S}: \mathcal{C}-\operatorname{Mod} \longrightarrow \mathcal{C}-\operatorname{Mod}, \quad V \mapsto V \circ \iota .
$$

From the usual tensor-hom adjunction, one can define a right adjoint functor $\mathrm{Q}$ to $\mathrm{S}$ called the coinduction functor. The main results of this paper are the following.

Theorem 1.3. Suppose that $k$ is a commutative ring, and $\mathcal{C}$ is the category $\mathrm{FI}_{G}$. Let $m \in$ $\mathrm{Ob}(\mathrm{C})$. Then $\mathrm{Q}\left(k \mathrm{C} e_{m}\right)$ is isomorphic to $k \mathrm{C} e_{m} \oplus k \mathrm{C} e_{m+1}$.

The proof of Theorem 1.3 will be given in Section 5 .

Denote by $q$ the number of elements of $\mathbb{F}$.

Theorem 1.4. Suppose that $k$ is a commutative ring, and $\mathcal{C}$ is the category VI. Let $m \in$ $\mathrm{Ob}(\mathrm{C})$. If $q$ is a unit of $k$, then $\mathrm{Q}\left(k \mathrm{C} e_{m}\right)$ contains a direct summand isomorphic to $k \mathrm{C} e_{m+1}$.

To prove Theorem 1.4, we construct a non-obvious surjective homomorphism from $\mathrm{Q}\left(k \mathrm{C} e_{m}\right)$ to $k \mathrm{C} e_{m+1}$. The details will be given in Section 6 .

1.3. Applications. Let us indicate quickly the use of the coinduction functor in this paper.

Suppose $\mathcal{C}$ is the category $\mathrm{FI}_{G}$ or VI. It is known that $\mathrm{S}(V)$ is projective if $V$ is a finitely generated projective $\mathcal{C}$-module. Hence, by the Eckman-Shapiro lemma, one has $\operatorname{Ext}_{\mathcal{C}}^{1}(\mathrm{~S}(V), W)=\operatorname{Ext}_{\mathcal{C}}^{1}(V, \mathrm{Q}(W))$ for any $\mathcal{C}$-modules $V$ and $W$. It follows that $\mathrm{Q}(W)$ is injective if $W$ is injective.

Suppose now that $k$ is a field of characteristic 0 . In this case, $\mathcal{C}$ is locally Noetherian. By reducing to finite categories, one can show that $k \mathcal{C}_{0}$ is an injective $\mathcal{C}$-module. It follows by induction, using Theorems 1.3 and 1.4, that $k \mathrm{C} e_{n}$ is injective for all $n \in \mathrm{Ob}(\mathrm{C})$. Hence, we deduce the following result.

Theorem 1.5. Suppose that $k$ is a field of characteristic 0 , and $\mathcal{C}$ is the category $\mathrm{FI}_{G}$ or VI. Let $V$ be a finitely generated projective $\mathcal{C}$-module. Then $V$ is an injective $\mathcal{C}$-module. 
We shall show, in Section 3 , that if $k$ is a field of positive characteristic and $\mathcal{C}$ is FI, the projective $\mathcal{C}$-module $k \mathfrak{C} e_{0}$ is not injective.

Corollary 1.6. Suppose that $k$ is a field of characteristic 0 , and $\mathcal{C}$ is the category $\mathrm{FI}_{G}$ or VI. Let $V$ be a finitely generated $\mathcal{C}$-module. Then $V$ has a finite projective resolution in the category $\mathcal{C}$-mod if and only if $V$ is a projective $\mathcal{C}$-module.

If $F$ is a nonzero finitely generated torsion-free $\mathcal{C}$-module, then there is a smallest $a \in \mathrm{Ob}(\mathcal{C})$ such that $F(a) \neq 0$, and it is easy to see that $\mathrm{S}^{a}(F)$ contains $k \mathcal{C} e_{0}$ as a $\mathcal{C}$-submodule. Since $k \mathrm{C} e_{0}$ is injective, it is a direct summand of $\mathrm{S}^{a}(F)$. By the adjunction of $\mathrm{S}$ and $\mathrm{Q}$, it follows that there exists a nonzero homomorphism from $F$ to $\mathrm{Q}^{a}\left(k \mathcal{C} e_{0}\right)$. Thus, when $\mathcal{C}$ is $\mathrm{FI}_{G}$, it follows from Theorem 1.3 that every nonzero finitely generated torsion-free $\mathcal{C}$-module has a nonzero homomorphism to a finitely generated projective $\mathcal{C}$-module. From this, it is not difficult to deduce the following result.

Theorem 1.7. Suppose that $k$ is a field of characteristic 0 , and $\mathcal{C}$ is the category $\mathrm{FI}_{G}$. Then one has the following.

(i) Any finitely generated injective $\mathcal{C}$-module is a direct sum of a finite dimensional injective $\mathcal{C}$-module and a finitely generated projective $\mathfrak{C}$-module.

(ii) Any finitely generated $\mathcal{C}$-module has a finite injective resolution in the category of finitely generated $\mathcal{C}$-modules.

The proofs of Theorems 1.5 and 1.7 will be given in Section 7. We do not know if Theorem 1.7 holds for the category VI.

Remark 1.8. Theorems 1.5 and 1.7 were first proved for the category FI by Sam and Snowden in [13]. Our proofs are new and independent of their results.

Remark 1.9. In [10], Kuhn studied the category of $\mathcal{C}$-modules where $\mathcal{C}$ is the category of finite dimensional $\mathbb{F}$-vector spaces with all linear maps as morphisms. He showed that if $k$ is a field and $q$ is a unit in $k$, the category of $\mathcal{C}$-modules is equivalent to the product over all $n \in \mathbb{Z}_{+}$of the categories of $\mathrm{GL}_{n}(\mathbb{F})$-modules; see [10, Theorem 1.1]. As a consequence, if $k$ is a field of characteristic 0 , the category of $\mathcal{C}$-modules is semisimple; see [10, Corollary 1.3]. In contrast, the categories $\mathrm{FI}_{G}$-Mod and VI-Mod do not have a similar decomposition, and Corollary 1.6 implies that the categories $\mathrm{FI}_{G}$-mod and VI-mod have infinite global dimension when characteristic of $k$ is 0 .

1.4. Representation stability. An upshot of Theorem 1.7 is an alternative proof of a key theorem of Church, Ellenberg, and Farb in their theory of representation stability. We shall discuss this in the more general situation of wreath product groups.

Suppose that $k$ is a splitting field for $G$ of characteristic 0 . Denote by $\operatorname{Irr}(G)$ the set of isomorphism classes of simple $k G$-modules, and $\chi_{1} \in \operatorname{Irr}(G)$ the trivial class. For each $n \in \mathbb{Z}_{+}$, the isomorphism classes of simple $k G \imath S_{n}$-modules are parametrized by partitionvalued functions $\underline{\lambda}$ on $\operatorname{Irr}(G)$ such that $|\underline{\lambda}|=n$, where

$$
|\underline{\lambda}|=\sum_{\chi \in \operatorname{Irr}(G)}|\underline{\lambda}(\chi)| .
$$

Suppose $\underline{\lambda}$ is any partition-valued function on $\operatorname{Irr}(G)$ and $n$ is any integer $\geqslant|\underline{\lambda}|+a$, where $a$ is the biggest part of $\underline{\lambda}\left(\chi_{1}\right)$. Following [15], we define the partition-valued function $\underline{\lambda}[n]$ on 
$\operatorname{Irr}(G)$ by

$$
\underline{\lambda}[n](\chi)= \begin{cases}\left(n-|\underline{\lambda}|, \underline{\lambda}\left(\chi_{1}\right)\right) & \text { if } \chi=\chi_{1}, \\ \underline{\lambda}(\chi) & \text { if } \chi \neq \chi_{1} .\end{cases}
$$

Let $L(\underline{\lambda})_{n}$ be a simple $k G \imath S_{n}$-module belonging to the isomorphism class corresponding to $\underline{\lambda}[n]$.

Suppose $V_{n}$ is a sequence of $k G 2 S_{n}$-modules equipped with linear maps $\phi_{n}: V_{n} \rightarrow V_{n+1}$. We say that $\left\{V_{n}\right\}$ is a consistent sequence if the following diagram commutes for each $g \in G \imath S_{n}$ :

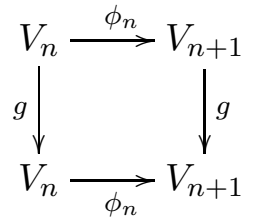

Here, $g$ acts on $V_{n+1}$ by its image under the standard inclusion $G$ 々 $S_{n} \hookrightarrow G$ २ $S_{n+1}$.

Definition 1.10. A consistent sequence $\left\{V_{n}\right\}$ of $k G\left\{S_{n}\right.$-modules is representation stable if there exists $N>0$ such that for each $n \geqslant N$, the following three conditions hold:

(RS1) Injectivity: The map $\phi_{n}: V_{n} \longrightarrow V_{n+1}$ is injective.

(RS2) Surjectivity: The span of the $G$ ? $S_{n+1}$-orbit of $\phi_{n}\left(V_{n}\right)$ is all of $V_{n+1}$.

(RS3) Multiplicities: There is a decomposition

$$
V_{n}=\bigoplus_{\underline{\lambda}} L(\underline{\lambda})_{n}^{\oplus c(\underline{\lambda})}
$$

where the multiplicities $0 \leqslant c(\underline{\lambda}) \leqslant \infty$ do not depend on $n$.

Remark 1.11. Our terminology of representation stable follows [6]. In [4] and [2], this is called uniformly representation stable.

Suppose $\mathcal{C}$ is $\mathrm{FI}_{G}$. Let $\left(\iota_{n}, c_{n}\right) \in \operatorname{Hom}_{\mathcal{C}}(n, n+1)$ be the morphism where $\iota_{n}:[n] \hookrightarrow[n+1]$ is the standard inclusion and $c_{n}:[n] \rightarrow G$ is the constant map whose image is the identity element of $G$. If $V$ is a $\mathcal{C}$-module, then $\{V(n)\}$ is a consistent sequence of $k G<S_{n}$-modules where the maps $\phi_{n}: V(n) \rightarrow V(n+1)$ are induced by the morphisms $\left(\iota_{n}, c_{n}\right)$. The following theorem was first proved in [2, Theorem 1.13] when $G$ is trivial.

Theorem 1.12 (Finite generation vs. representation stability). Suppose that $k$ is a splitting field for $G$ of characteristic 0 . Let $V$ be a $\mathrm{FI}_{G}$-module. Then $V$ is finitely generated if and only if $\{V(n)\}$ is a representation stable sequence of $k G<S_{n}$-modules with $\operatorname{dim}_{k} V(n)<\infty$ for each $n$.

It is easy to see that $V$ is finitely generated if and only if condition (RS2) holds and $\operatorname{dim}_{k} V(n)<\infty$ for each $n$; see [7, Proposition 5.2]. Suppose that $V$ is a finitely generated C-module. Then condition (RS1) is a simple consequence of the fact that $V$ is Noetherian; see 7. Proposition 5.1]. The real task is to prove that condition (RS3) holds. But by Theorem 1.7. it suffices to verify condition (RS3) for finitely generated projective $\mathcal{C}$-modules, and this is easily accomplished by Pieri's formula. We shall give the details of the proof of Theorem 1.12 in Section 8 . 
1.5. Bibliographical remarks. Church and Farb introduced the notion of representation stability for various families of groups in [4. The connection of representation stability for sequences of $S_{n}$-representations to FI-modules was subsequently made by Church, Ellenberg, and Farb in 2]; their paper contains many interesting examples of representation stable sequences in algebra, geometry and topology. It was also proved in [2, Theorem 1.3] that FI is locally Noetherian over any field $k$ of characteristic 0 ; this result was later extended to an arbitrary Noetherian ring $k$ by Church, Ellenberg, Farb, and Nagpal in [3, Theorem A].

In [18, Wilson defined and studied $\mathrm{FI}_{\mathcal{W}}$-modules associated to the Weyl groups of type $\mathrm{B} / \mathrm{C}$ and type D. In particular, she gave a proof of the analogue of Theorem 1.12 in these cases along the same lines as the proof in [2]. Using [3, Theorem A], she proved that $\mathrm{FI}_{\mathcal{W}}$ is locally Noetherian over any Noetherian ring $k$. In the type $\mathrm{B} / \mathrm{C}$ case, the category $\mathrm{FI}_{\mathcal{W}}$ is same as the category $\mathrm{FI}_{G}$ for the group $G$ of order 2 .

From a different point of view, Snowden [16, Theorem 2.3] also proved that FI is locally Noetherian over any field $k$ of characteristic 0 . In fact, he proved this for any twisted commutative algebra finitely generated in order 1. Theorems 1.5 and 1.7 for the category FI were proved by Sam and Snowden in [13, Corollary 4.2.5], [13, Theorem 4.3.1], and [13, Theorem 4.3.4]. The strategy of their proofs is to pass to the Serre quotient of the category of finitely generated FI-modules by the subcategory of finite dimensional FI-modules. Using their results, Sam and Snowden deduced a formula for the character polynomial of a finitely generated FI-module over a field of characteristic 0 .

In [7, we gave a simple proof that $\mathrm{FI}_{G}$ and VI are locally Noetherian over any field $k$ of characteristic 0 (which is sufficient for the present paper); a similar proof for FI and VI was also obtained by Putman (unpublished). At about the same time, using Gröbner basis methods, Putman and Sam [12, Theorem A] proved that VI is locally Noetherian when $\mathbb{F}$ is any finite ring and $k$ is any Noetherian ring, and Sam and Snowden [14, Theorem 10.1.2] proved that $\mathrm{FI}_{G}$ is locally Noetherian for any Noetherian ring $k$. In [15, Theorem 1.2.4], Sam and Snowden proved that if $k$ is a field in which the order of $G$ is invertible, then representations of $\mathrm{FI}_{G}$ are in fact equivalent to representations of $\mathrm{FI} \times \mathrm{FB}^{a}$, where $\mathrm{FB}$ is the groupoid of finite sets and $a$ is the number of non-trivial irreducible representations of $G$. Using this, they proved a wreath-product version of Murnaghan's stability theorem [15, Theorem 5.2.1].

It was first observed by Church, Ellenberg, Farb, and Nagpal in [3, Proposition 2.12] that the functor $\mathrm{S}$ for the category FI has the property that $\mathrm{S}(V)$ is projective whenever $V$ is a finitely generated projective FI-module. A more precise version of this property plays a crucial role in their paper. In [8, Section 5], we showed that the functor S for many other categories have this property, including $\mathrm{FI}_{G}$ and VI. To the best of our knowledge, no one has studied the coinduction functor $Q$, even in the case of FI.

\section{Preliminaries}

2.1. Notations and terminology. Recall that an EI category is a category in which every endomorphism is an isomorphism (see [5]). Throughout this paper, we shall denote by $\mathcal{C}$ an EI category satisfying the following conditions:

- $\mathrm{Ob}(\mathrm{C})=\mathbb{Z}_{+}$;

- $\mathrm{C}(m, n)$ is an empty set if $m>n$; 
- $\mathcal{C}(m, n)$ is a nonempty finite set if $m \leqslant n$;

- for $m \leqslant l \leqslant n$, the composition map $\mathcal{C}(l, n) \times \mathcal{C}(m, l) \rightarrow \mathcal{C}(m, n)$ is surjective.

Suppose $n \in \mathrm{Ob}(\mathcal{C})$. We shall use the following notations:

- $G_{n}$ denotes the group $\mathcal{C}(n, n)$;

- $\mathcal{C}_{n}$ denotes the full subcategory of $\mathcal{C}$ with $\mathrm{Ob}\left(\mathfrak{C}_{n}\right)=\{0,1, \ldots, n\}$.

Suppose that $k$ is a commutative ring and $V$ is a $\mathcal{C}$-module. For $n \in \mathrm{Ob}(\mathcal{C})$ and $v \in V(n)$, we say that $v$ has degree $n$, and write $\operatorname{deg}(v)$ for the degree of $v$. By a set of generators of $V$, we shall always mean a subset $S$ of $\bigcup_{n \in \mathrm{Ob}(\mathrm{e})} V(n)$ such that the only submodule of $V$ containing $S$ is $V$ itself. We say that $V$ is generated in degrees $\leqslant n$ if $V$ has a set $S$ of generators such that the degree of each element of $S$ is at most $n$. We say that $V$ is finitely generated if $V$ has a finite set of generators. We say that $V$ is Noetherian if every submodule of $V$ is finitely generated. We say that $\mathcal{C}$ is locally Noetherian if every finitely generated $\mathrm{C}$-module is Noetherian.

2.2. Baer's criterion. We omit the proof of the following lemma, which is standard (see [17, page 39]).

Lemma 2.1 (Baer's criterion). Suppose that $k$ is a commutative ring, and $V \in \mathcal{C}$-Mod. Suppose that for all $n \in \mathrm{Ob}(\mathcal{C})$ and for all $\mathcal{C}$-submodule $U$ of $k \mathfrak{C} e_{n}$, every homomorphism $U \rightarrow V$ can be extended to a homomorphism $k \mathrm{C}_{n} \rightarrow V$. Then $V$ is injective in $\mathrm{C}$-Mod.

The following corollary is immediate.

Corollary 2.2. Suppose that $k$ is a commutative ring and $\mathcal{C}$ is locally Noetherian. Let $V \in \mathcal{C}$-mod. Then $V$ is injective in $\mathcal{C}$-Mod if and only if $V$ is injective in $\mathcal{C}$-mod.

2.3. Projective resolutions. Suppose that $k$ is a commutative ring. For any $n \in \mathrm{Ob}(\mathcal{C})$, the $\mathrm{C}$-module $k \mathrm{C} e_{n}$ is clearly projective. Suppose $V$ is a $\mathcal{C}$-module and $S$ is a set of generators of $V$. Then there is a surjective homomorphism $\bigoplus_{s \in S} k \mathcal{C} e_{\operatorname{deg}(s)} \rightarrow V$ whose restriction to the direct summand corresponding to $s$ is $\alpha \mapsto \alpha s$. Hence, any $\mathcal{C}$-module $V$ has a projective resolution

$$
\cdots \rightarrow P^{-2} \rightarrow P^{-1} \rightarrow P^{0} \rightarrow V \rightarrow 0
$$

such that each $P^{-i}$ is a direct sum of projective $\mathcal{C}$-modules of the form $k \mathcal{C} e_{n}$ where $n \in \mathrm{Ob}(\mathcal{C})$.

2.4. Restriction to $\mathcal{C}_{n}$. Suppose that $k$ is a commutative ring. Let $n \in \mathrm{Ob}(\mathcal{C})$. Recall that $\mathcal{C}_{n}$ denotes the full subcategory of $\mathcal{C}$ with $\mathrm{Ob}\left(\mathcal{C}_{n}\right)=\{0,1, \ldots, n\}$; see Subsection 2.1. Denote by $\jmath: \mathcal{C}_{n} \hookrightarrow \mathcal{C}$ the inclusion functor. We have the pullback functor

$$
\jmath^{*}: \mathcal{C}-\operatorname{Mod} \longrightarrow \mathcal{C}_{n} \text {-Mod, } \quad V \mapsto V \circ \jmath .
$$

We also have the pushforward functor

$$
\jmath_{*}: \mathcal{C}_{n}-\operatorname{Mod} \longrightarrow \mathcal{C}-\operatorname{Mod}
$$

which regards a $\mathcal{C}_{n}$-module as a $\mathcal{C}$-module in the obvious way. The pushforward functor $\jmath_{*}$ is a right adjoint functor to the pullback functor $\jmath^{*}$.

Lemma 2.3. Suppose that $k$ is a commutative ring. Let $n \in \mathrm{Ob}(\mathcal{C})$ and denote by $\jmath: \mathfrak{C}_{n} \hookrightarrow \mathcal{C}$ the inclusion functor. For any $V \in \mathcal{C}$-Mod and $W \in \mathcal{C}_{n}$-Mod, one has

$$
\operatorname{Ext}_{\mathcal{C}_{n}}^{i}\left(\jmath^{*}(V), W\right)=\operatorname{Ext}_{\mathfrak{C}}^{i}\left(V, \jmath_{*}(W)\right)
$$


for all $i \geqslant 1$.

Proof. Observe that $\jmath^{*}\left(k \mathcal{C} e_{m}\right)$ is a projective $\mathcal{C}_{n}$-module for all $m \in \mathrm{Ob}(\mathcal{C})$. Thus, the required result follows from the Eckmann-Shapiro lemma.

Lemma 2.4. Suppose that $k$ is a commutative ring and $\mathcal{C}$ is locally Noetherian. Let $V, W \in$ $\mathcal{C}$-mod. Then there exists $N \in \mathrm{Ob}(\mathcal{C})$ such that for all $n \geqslant N$, one has

$$
\operatorname{Ext}_{\mathcal{C}}^{1}(V, W)=\operatorname{Ext}_{\mathcal{C}_{n}}^{1}\left(\jmath^{*}(V), \jmath^{*}(W)\right)
$$

where $\mathrm{\jmath}: \mathrm{C}_{n} \hookrightarrow \mathcal{C}$ is the inclusion functor.

Proof. Since $\mathcal{C}$ is locally Noetherian, there exists a projective resolution

$$
\cdots \rightarrow P^{-2} \rightarrow P^{-1} \rightarrow P^{0} \rightarrow V \rightarrow 0
$$

such that each $P^{-i}$ is a finitely generated projective $\mathcal{C}$-module. Thus, there exists $N \in \mathrm{Ob}(\mathcal{C})$ such that $P^{-2}$ and $P^{-1}$ are both generated in degrees $\leqslant N$.

Suppose $n \geqslant N$. Let $U$ be the submodule $\bigoplus_{m>n} W(m)$ of $W$. We have a short exact sequence

$$
0 \longrightarrow U \longrightarrow W \longrightarrow \jmath_{*}\left(\jmath^{*}(W)\right) \longrightarrow 0,
$$

and hence a long exact sequence

$$
\cdots \rightarrow \operatorname{Ext}_{\mathcal{C}}^{1}(V, U) \rightarrow \operatorname{Ext}_{\mathcal{C}}^{1}(V, W) \rightarrow \operatorname{Ext}_{\mathcal{C}}^{1}\left(V, \jmath_{*}\left(\jmath^{*}(W)\right)\right) \rightarrow \operatorname{Ext}_{\mathcal{C}}^{2}(V, W) \rightarrow \cdots
$$

But, for $i=1,2$, one has $\operatorname{Hom}_{\mathcal{C}}\left(P^{-i}, U\right)=0$ and so $\operatorname{Ext}_{\mathcal{C}}^{i}(V, U)=0$. It follows that

$$
\operatorname{Ext}_{\mathcal{C}}^{1}(V, W)=\operatorname{Ext}_{\mathcal{C}}^{1}\left(V, \jmath_{*}\left(\jmath^{*}(W)\right)\right)=\operatorname{Ext}_{\mathcal{C}_{n}}^{1}\left(\jmath^{*}(V), \jmath^{*}(W)\right),
$$

using Lemma 2.3.

2.5. Injective resolutions of finite dimensional modules. Suppose that $k$ is a field. We denote by $\mathrm{D}$ the standard duality functor $\operatorname{Hom}_{k}(-, k)$ between the categories $\mathcal{C}_{n}$-mod and $\mathcal{C}_{n}^{\text {op }}$-mod. Any finite dimensional injective $\mathcal{C}_{n}$-module is isomorphic to $\mathrm{D}(P)$ for some finite dimensional projective $\mathcal{C}_{n}^{\mathrm{op}}$-module $P$.

Lemma 2.5. Suppose $k$ is a field. If $W$ is a finite dimensional injective $\mathcal{C}_{n}$-module for some $n \in \mathrm{Ob}(\mathrm{C})$, then $\jmath_{*}(W)$ is a finite dimensional injective $\mathcal{C}$-module, where $\jmath: \mathfrak{C}_{n} \hookrightarrow \mathcal{C}$.

Proof. It is obvious that $\jmath_{*}(W)$ is finite dimensional. The injectivity of $\jmath_{*}(W)$ follows from Lemma 2.3 .

Lemma 2.6. Suppose $k$ is a field of characteristic 0. Then every finite dimensional $\mathrm{C}$-module $V$ has a finite injective resolution

$$
0 \rightarrow V \rightarrow I^{0} \rightarrow I^{1} \rightarrow \cdots I^{m} \rightarrow 0
$$

where $I^{r}$ is finite dimensional for each $r \in\{0,1, \ldots, m\}$.

Proof. Choose $n \in \mathrm{Ob}(\mathcal{C})$ such that $V=\jmath_{*}\left(\jmath^{*}(V)\right)$ where $\jmath: \mathcal{C}_{n} \hookrightarrow \mathcal{C}$. It is easy to see that any finite dimensional $\mathcal{C}_{n}^{\text {op }}$-module has a finite projective resolution in the category $\mathcal{C}_{n}^{\text {op }}$-mod. Using the functor $\mathrm{D}$, we deduce that $\jmath^{*}(V)$ has a finite injective resolution in the category $\mathcal{C}_{n}$-mod. By Lemma 2.5, applying the functor $\jmath_{*}$ to this resolution gives a resolution of $V$ of the required form. 
Remark 2.7. Suppose $k$ is a field, and $V$ is a finite dimensional injective $\mathcal{C}$-module. It is easy to see that if $n \in \mathrm{Ob}(\mathcal{C})$ and $V=\jmath_{*}\left(\jmath^{*}(V)\right)$, where $\jmath: \mathcal{C}_{n} \hookrightarrow \mathcal{C}$ is the inclusion functor, then $\jmath^{*}(V)$ is an injective $\mathcal{C}_{n}$-module.

\section{InJeCtivity of $k \mathrm{C} e_{0}$}

3.1. Field of characteristic $\mathbf{0}$. We say that the category $\mathcal{C}$ satisfies the transitivity condition if for all $n \in \mathrm{Ob}(\mathcal{C})$, the action of $G_{n+1}$ on $\mathcal{C}(n, n+1)$ is transitive. (Recall that $G_{n}$ denotes the group $\mathcal{C}(n, n)$; see Subsection 2.1.)

Lemma 3.1. Suppose that $k$ is a field of characteristic 0. If $\mathrm{e}$ satisfies the transitivity condition, and 0 is an initial object of $\mathcal{C}$, then $\mathrm{K}_{n} e_{0}$ is an injective $\mathcal{C}_{n}$-module for all $n \in$ $\mathrm{Ob}(\mathrm{e})$.

Proof. It suffices to prove that $\operatorname{Ext}_{\mathcal{C}_{n}}^{1}\left(k G_{m}, k \mathcal{C}_{n} e_{0}\right)=0$ for all $m \in \mathrm{Ob}\left(\mathcal{C}_{n}\right)$. This is clear when $m=n$ for $k G_{n}$ is a projective $\mathcal{C}_{n}$-module.

Suppose $m<n$. Let $P=k \mathrm{C}_{n} e_{m}$ and let $U$ be the submodule $\bigoplus_{l>m} P(l)$ of $P$. We have a short exact sequence

$$
0 \longrightarrow U \longrightarrow P \longrightarrow k G_{m} \longrightarrow 0
$$

and hence a long exact sequence

$$
\begin{aligned}
0 \rightarrow \operatorname{Hom}_{\mathcal{C}_{n}}\left(k G_{m}, k \mathcal{C}_{n} e_{0}\right) \rightarrow \operatorname{Hom}_{\mathcal{C}_{n}}( & P, \\
& \left.k \mathcal{C}_{n} e_{0}\right) \rightarrow \operatorname{Hom}_{\mathfrak{C}_{n}}\left(U, k \mathcal{C}_{n} e_{0}\right) \\
& \rightarrow \operatorname{Ext}_{\mathfrak{C}_{n}}^{1}\left(k G_{m}, k \mathcal{C}_{n} e_{0}\right) \rightarrow \operatorname{Ext}_{\mathcal{C}_{n}}^{1}\left(P, k \mathcal{C}_{n} e_{0}\right) \rightarrow \cdots
\end{aligned}
$$

Since $m<n$, one has $\operatorname{Hom}_{\mathcal{C}_{n}}\left(k G_{m}, k \mathcal{C}_{n} e_{0}\right)=0$. Since $P$ is projective, $\operatorname{Ext}_{\mathcal{C}_{n}}^{1}\left(P, k \mathcal{C}_{n} e_{0}\right)=0$. Note that

$$
\operatorname{dim}_{k} \operatorname{Hom}_{k \mathfrak{C}_{n}}\left(P, k \mathfrak{C}_{n} e_{0}\right)=\operatorname{dim}_{k} k \mathfrak{C}_{n}(0, m)=1 .
$$

Since $U$ is generated by $U(m+1)$, and $G_{m+1}$ acts transitively on $U(m+1)$, one has

$$
\operatorname{dim}_{k} \operatorname{Hom}_{k \mathfrak{C}_{n}}\left(U, k \mathcal{C}_{n} e_{0}\right) \leqslant \operatorname{dim}_{k} \operatorname{Hom}_{k G_{m+1}}\left(U(m+1), k \mathfrak{C}_{n}(0, m+1)\right) \leqslant 1 .
$$

Hence, we must have $\operatorname{Ext}_{\mathcal{C}_{n}}^{1}\left(k G_{m}, k \mathcal{C}_{n} e_{0}\right)=0$

Corollary 3.2. Suppose that $k$ is a field of characteristic 0. If $\mathrm{C}$ is locally Noetherian, satisfies the transitivity condition, and 0 is an initial object of $\mathcal{C}$, then $k \mathrm{Ce}_{0}$ is an injective e-module.

Proof. By Corollary 2.2, it suffices to show that $k \mathcal{C}_{0}$ is injective in $\mathcal{C}$-mod. By Lemma 2.4 this follows from injectivity of $k \mathrm{C}_{n} e_{0}$ which is proved in Lemma 3.1 .

3.2. Field of characteristic $p$. Let us show that Corollary 3.2 is false when $k$ is a field of characteristic $p>0$ and $\mathcal{C}$ is the category FI.

For any $n \geqslant p$, we have a right action of the symmetric group $S_{p}$ on $\mathcal{C}(p, n)$. Let $U(n)$ be the subspace of $S_{p}$-invariant elements in $k \mathrm{C}(p, n)$, and let $U=\bigoplus_{n \geqslant p} U(n)$. Then $U$ is a submodule of $k \mathrm{C} e_{p}$. Now let $\mathcal{J}(n)$ be the set of $S_{p}$-orbits in $\mathcal{C}(p, n)$. For each orbit $J \in \mathcal{J}(n)$, let $\xi_{J} \in U(n)$ be the sum of all the $p$ ! elements in $J$. Then the collection of $\xi_{J}$ for $J \in \mathcal{J}(n)$ is a basis for $U(n)$. There is a homomorphism $f: U \rightarrow k \bigodot e_{0}$ such that $f\left(\xi_{J}\right)$ is the unique element of $\mathcal{C}(0, n)$ if $J \in \mathcal{J}(n)$. We define a submodule $W$ of $k \mathfrak{C} e_{0} \oplus k \mathfrak{C} e_{p}$ as follows. For 
any $n \geqslant p$, let $W(n)$ be the set of all elements in $k \mathrm{C}(0, n) \oplus U(n)$ of the form $f(\xi)+\xi$ for $\xi \in U(n)$.

Let $V=\left(k \mathcal{C} e_{0} \oplus k \mathcal{C} e_{p}\right) / W$, and let $\pi: k \bigodot e_{0} \oplus k \bigodot e_{p} \rightarrow V$ be the canonical projection. Denote by $i: k \mathrm{Ce}_{0} \rightarrow V$ the restriction of $\pi$ to $k \mathrm{Ce}_{0}$. It is clear that $i$ is a monomorphism. We claim that the short exact sequence

$$
0 \longrightarrow k \mathrm{C} e_{0} \stackrel{i}{\longrightarrow} V \longrightarrow V / i\left(k \mathrm{C} e_{0}\right) \longrightarrow 0
$$

does not split. Indeed, any homomorphism $k \mathcal{C} e_{0} \oplus k \mathcal{C} e_{p} \rightarrow k \mathcal{C} e_{0}$ whose restriction to $k \mathcal{C} e_{0}$ is the identity map cannot vanish identically on $W$, for any homomorphism $k \mathcal{C} e_{p} \rightarrow k \mathcal{C} e_{0}$ must vanish identically on $U$.

\section{RESTRICTION AND COINDUCTION ALONG GENETIC FUNCTORS}

Throughout this section, $k$ denotes any commutative ring.

4.1. Restriction and coinduction. A coinduction functor can be defined whenever one has a subring of a ring, and it is a right adjoint functor to the restriction functor; we refer the reader to [1, Section 2.8] for a clear exposition on the definition and basic properties of the coinduction functor in such a general setting. In this section, we begin our study of the coinduction functor in our special setting, where the pair of subring and the ring are isomorphic.

Let $\iota: \mathcal{C} \rightarrow \mathcal{C}$ be a faithful functor such that $\iota(n)=n+1$ for all $n \in \mathrm{Ob}(\mathcal{C})$. We define the restriction functor $\mathrm{S}: \mathcal{C}-\operatorname{Mod} \longrightarrow \mathcal{C}-\operatorname{Mod}$ by $\mathrm{S}(V)=V \circ \iota$ for all $V \in \mathcal{C}$-Mod; thus, $\mathrm{S}(V)(n)=V(n+1)$.

Definition 4.1. Suppose $V \in \mathcal{C}$-Mod. We define $\mathrm{Q}(V) \in \mathcal{C}$-Mod by

$$
\mathrm{Q}(V)(n)=\operatorname{Hom}_{\mathcal{C}}\left(\mathrm{S}\left(k \mathcal{C} e_{n}\right), V\right) \quad \text { for each } n \in \mathrm{Ob}(\mathcal{C}) \text {. }
$$

We call $\mathrm{Q}: \mathcal{C}-\operatorname{Mod} \longrightarrow \mathcal{C}-\operatorname{Mod}$ the coinduction functor.

Observe that any $\alpha \in \mathcal{C}(m, n)$ defines a $\mathcal{C}$-module homomorphism

$$
\mathrm{S}\left(k \mathrm{C} e_{n}\right) \longrightarrow \mathrm{S}\left(k \mathrm{C} e_{m}\right), \quad \gamma \mapsto \gamma \alpha .
$$

The $\mathcal{C}$-module structure on $\mathrm{Q}(V)$ is defined in the natural way as follows: if $\alpha \in \mathcal{C}(m, n)$ and $\varrho \in \mathrm{Q}(V)(m)$, then $\alpha(\varrho) \in \mathrm{Q}(V)(n)$ is the $\mathcal{C}$-module homomorphism

$$
\mathrm{S}\left(k \mathrm{C} e_{n}\right) \longrightarrow V, \quad \gamma \mapsto \varrho(\gamma \alpha) .
$$

Lemma 4.2. The functor $\mathrm{Q}$ is right adjoint to the functor $\mathrm{S}$.

Proof. Let

$$
M=\bigoplus_{\substack{m \geqslant 0 \\ n \geqslant 1}} k \mathrm{e}(m, n) .
$$

We have a $k \mathcal{C}$-bimodule structure on $M$ defined by

$$
\alpha \cdot \gamma=\iota(\alpha) \gamma, \quad \gamma \cdot \alpha=\gamma \alpha,
$$

for $\alpha \in k \mathrm{C}$ and $\gamma \in M$. By the tensor-hom adjunction, one has

$$
\operatorname{Hom}_{k \mathfrak{C}}\left(M \otimes_{k \mathrm{e}} V, W\right)=\operatorname{Hom}_{k \mathrm{e}}\left(V, \operatorname{Hom}_{k \mathrm{e}}(M, W)\right)
$$


for any $V, W \in \mathcal{C}$-Mod.

The $k \mathcal{C}$-module homomorphism

$$
M \otimes_{k \mathcal{C}} V \longrightarrow \mathrm{S}(V), \quad \gamma \otimes v \mapsto \gamma v
$$

has an inverse defined on $\mathrm{S}(V)(n)$ by $v \mapsto e_{n+1} \otimes v$, for each $n \in \mathrm{Ob}(\mathcal{C})$. Hence, $M \otimes_{k \mathcal{C}} V$ is isomorphic to $\mathrm{S}(V)$.

On the other hand, there is a $k \mathfrak{C}$-module direct sum decomposition

$$
M=\bigoplus_{m \geqslant 0} \mathrm{~S}\left(k \mathcal{C} e_{m}\right)
$$

so

$$
\operatorname{Hom}_{k \mathfrak{C}}(M, W)=\prod_{m \geqslant 0} \operatorname{Hom}_{\mathcal{C}}\left(\mathrm{S}\left(k \mathcal{C} e_{m}\right), W\right) .
$$

But since $V$ is a graded $k \mathfrak{C}$-module, the image of any $k \mathfrak{C}$-module homomorphism from $V$ to $\operatorname{Hom}_{k \mathcal{C}}(M, W)$ lies in $\mathrm{Q}(W)$. It follows that

$$
\operatorname{Hom}_{k \mathcal{C}}\left(V, \operatorname{Hom}_{k \mathfrak{C}}(M, W)\right)=\operatorname{Hom}_{\mathcal{C}}(V, \mathrm{Q}(W)) .
$$

Thus, $\operatorname{Hom}_{\mathcal{C}}(\mathrm{S}(V), W)=\operatorname{Hom}_{\mathcal{C}}(V, \mathrm{Q}(W))$.

Following [8], we call $\iota$ a genetic functor if, for each $n \in \mathrm{Ob}(\mathcal{C})$, the $\mathcal{C}$-module $\mathrm{S}\left(k \mathcal{C} e_{n}\right)$ is projective and generated in degrees $\leqslant n$.

Lemma 4.3. Suppose $\iota$ is a genetic functor. Then for any $V, W \in \mathcal{C}-\mathrm{Mod}$, one has

$$
\operatorname{Ext}_{\mathcal{C}}^{i}(\mathrm{~S}(V), W)=\operatorname{Ext}_{\mathcal{C}}^{i}(V, \mathrm{Q}(W))
$$

for all $i \geqslant 1$.

Proof. This is immediate from Lemma 4.2 and the Eckmann-Shapiro lemma.

Remark 4.4. The condition in Lemma 4.3 that $\iota$ is a genetic functor can be weakened. Indeed, we only need to use the property that for each $n \in \mathrm{Ob}(\mathcal{C})$, the $\mathcal{C}$-module $\mathrm{S}\left(k \mathcal{C} e_{n}\right)$ is projective.

4.2. Genetic functors for $\mathrm{FI}_{G}$ and VI. Suppose $\mathcal{C}$ is $\mathrm{FI}_{G}$ or VI. There is a natural monoidal structure $\odot$ on $\mathcal{C}$ such that $m \odot n=m+n$ for all $m, n \in \mathrm{Ob}(\mathcal{C})$. Let us recall this monoidal structure.

Case 1: Suppose $\mathcal{C}$ is $\mathrm{FI}_{G}$. For any $\left(f_{1}, c_{1}\right) \in \mathcal{C}\left(m_{1}, n_{1}\right)$ and $\left(f_{2}, c_{2}\right) \in \mathcal{C}\left(m_{2}, n_{2}\right)$, we define $\left(f_{1}, c_{1}\right) \odot\left(f_{2}, c_{2}\right)$ to be the morphism $(f, c) \in \mathcal{C}\left(m_{1}+m_{2}, n_{1}+n_{2}\right)$ where

$$
f(t)= \begin{cases}f_{1}(t) & \text { if } t \leqslant m_{1}, \\ f_{2}\left(t-m_{1}\right)+n_{1} & \text { if } t>m_{1} .\end{cases}
$$

and

$$
c(t)= \begin{cases}c_{1}(t) & \text { if } r \leqslant m_{1}, \\ c_{2}\left(t-m_{1}\right) & \text { if } t>m_{1} .\end{cases}
$$

Case 2: Suppose $\mathcal{C}$ is VI. For any $f_{1} \in \mathcal{C}\left(m_{1}, n_{1}\right)$ and $f_{2} \in \mathcal{C}\left(m_{2}, n_{2}\right)$, we define $f_{1} \odot f_{2} \in$ $\mathrm{e}\left(m_{1}+m_{2}, n_{1}+n_{2}\right)$ by

$$
f_{1} \odot f_{2}=f_{1} \oplus f_{2}: \mathbb{F}^{m_{1}} \oplus \mathbb{F}^{m_{2}} \longrightarrow \mathbb{F}^{n_{1}} \oplus \mathbb{F}^{n_{2}} .
$$


In both cases, we let $\iota: \mathcal{C} \rightarrow \mathcal{C}$ be the functor defined by (1.1). It is clear that $\iota$ is faithful. In the next two sections, we shall briefly recall the proof that $\iota$ is a genetic functor, and examine the structure of $\mathrm{Q}\left(k \mathcal{C} e_{m}\right)$ for each $m \in \mathrm{Ob}(\mathcal{C})$.

\section{Structure of $\mathrm{Q}\left(k \mathcal{C} e_{m}\right)$ when $\mathcal{C}$ is $\mathrm{FI}_{G}$}

Throughout this section, $k$ denotes any commutative ring.

5.1. Structure of $\mathrm{S}\left(k \mathcal{C} e_{n}\right)$. Suppose that $\mathcal{C}$ is $\mathrm{FI}_{G}$. Let $n \in \mathrm{Ob}(\mathcal{C})$. We now recall the structure of $\mathrm{S}\left(k \mathrm{C} e_{n}\right)$.

Denote by $e$ the identity element of $G$, and define the morphisms

$$
\left(f_{n}, c_{n}\right) \in \mathcal{C}(n, n+1) \quad \text { and } \quad\left(f_{n, r, g}, c_{n, r, g}\right) \in \mathcal{C}(n, n) \quad \text { for } r \in[n], g \in G,
$$

by

$$
\begin{aligned}
f_{n}(t) & =t+1, \\
c_{n}(t) & =e, \\
f_{n, r, g}(t) & = \begin{cases}t+1 & \text { if } t<r, \\
1 & \text { if } t=r, \\
t & \text { if } t>r,\end{cases} \\
c_{n, r, g}(t) & = \begin{cases}e & \text { if } t \neq r, \\
g & \text { if } t=r,\end{cases}
\end{aligned}
$$

for $t \in[n]$.

Now, for any $l \in \mathrm{Ob}(\mathcal{C}), r \in[n], g \in G$, define the maps

$$
\begin{aligned}
\Phi_{n, 0}: \mathcal{C}(n, l) & \longrightarrow \mathcal{C}(n, l+1), \quad \alpha \mapsto \iota(\alpha) \circ\left(f_{n}, c_{n}\right) ; \\
\Phi_{n, r, g}: \mathcal{C}(n-1, l) & \longrightarrow \mathcal{C}(n, l+1), \quad \alpha \mapsto \iota(\alpha) \circ\left(f_{n, r, g}, c_{n, r, g}\right) .
\end{aligned}
$$

We may extend these maps linearly to $\mathcal{C}$-module homomorphisms

$$
\Phi_{n, 0}: k \mathrm{C} e_{n} \longrightarrow \mathrm{S}\left(k \mathrm{C} e_{n}\right) \quad \text { and } \quad \Phi_{n, r, g}: k \mathcal{C} e_{n-1} \longrightarrow \mathrm{S}\left(k \mathrm{C} e_{n}\right) .
$$

Let

$$
\Phi_{n}: k \bigodot e_{n} \oplus\left(\bigoplus_{r \in[n], g \in G} k \mathrm{C} e_{n-1}\right) \longrightarrow \mathrm{S}\left(k \mathcal{C} e_{n}\right)
$$

be the $\mathcal{C}$-module homomorphism whose restriction to $k \mathcal{C} e_{n}$ is $\Phi_{n, 0}$ and whose restriction to the direct summand $k e_{n-1}$ indexed by $r \in[n], g \in G$ is $\Phi_{n, r, g}$. It is straightforward to verify that $\Phi_{n}$ is an isomorphism (see [8, Section 5]). Thus, $\iota$ is a genetic functor.

5.2. Preliminary discussion of $\mathrm{Q}\left(k \mathcal{C} e_{m}\right)$. We retain the notations of subsection 5.1. Let $m, n \in \mathrm{Ob}(\mathcal{C})$. By the isomorphism (5.1), one has the identification

$$
\mathrm{Q}\left(k \mathcal{C} e_{m}\right)(n)=\operatorname{Hom}_{\mathcal{C}}\left(k \mathcal{C} e_{n}, k \mathcal{C} e_{m}\right) \oplus\left(\bigoplus_{r \in[n], g \in G} \operatorname{Hom}_{\mathcal{C}}\left(k \mathcal{C} e_{n-1}, k \mathcal{C} e_{m}\right)\right)
$$


Denote by

$$
\begin{aligned}
\Psi_{n, 0}: k \mathcal{C}(m, n) & \longrightarrow \mathrm{Q}\left(k \mathcal{C} e_{m}\right)(n), \\
\Psi_{n, r, g}: k \mathcal{C}(m, n-1) & \longrightarrow \mathrm{Q}\left(k \mathcal{C} e_{m}\right)(n)
\end{aligned}
$$

the linear maps where $\Psi_{n, 0}$ is the natural bijection of $k \mathrm{C}(m, n)$ with the direct summand $\operatorname{Hom}_{\mathcal{C}}\left(k \mathcal{C} e_{n}, k \mathrm{C} e_{m}\right)$ of $\mathrm{Q}\left(k \mathcal{C} e_{m}\right)(n)$, and $\Psi_{n, r, g}$ is the natural bijection of $k \mathrm{C}(m, n-1)$ with the direct summand $\operatorname{Hom}_{\mathcal{C}}\left(k \mathrm{C} e_{n-1}, k \mathcal{C} e_{m}\right)$ of $\mathrm{Q}\left(k \mathcal{C} e_{m}\right)(n)$ indexed by $r \in[n], g \in G$. We have a linear bijection

$$
\Psi_{n}: k \mathfrak{C}(m, n) \oplus\left(\bigoplus_{r \in[n], g \in G} k \mathfrak{C}(m, n-1)\right) \longrightarrow \mathrm{Q}\left(k \mathcal{C} e_{m}\right)(n)
$$

whose restriction to $k \mathrm{e}(m, n)$ is $\Psi_{n, 0}$ and whose restriction to the direct summand $k \mathrm{C}(m, n-1)$ indexed by $r \in[n], g \in G$ is $\Psi_{n, r, g}$.

The next lemma describes the $\mathcal{C}$-module structure of $\mathrm{Q}\left(k \mathcal{C} e_{m}\right)$ in terms of the identifications $\Psi_{n}$ for $n \in \mathrm{Ob}(\mathcal{C})$. We shall use the following notations. For any $l \geqslant 1$ and $r \in[l]$, let $\partial_{r}:[l] \backslash\{r\} \rightarrow[l-1]$ be the unique nondecreasing bijection. If $\alpha=(f, c) \in \mathcal{C}(n, l)$, $r \in[l] \backslash \operatorname{Im}(f)$, and $s \in[n]$, we let

$$
\begin{aligned}
\partial_{r} \alpha & =\left(\partial_{r} \circ f, c\right) \in \mathcal{C}(n, l-1), \\
\alpha_{s} & =\left(\partial_{f(s)} \circ f \circ \partial_{s}^{-1}, c \circ \partial_{s}^{-1}\right) \in \mathcal{C}(n-1, l-1),
\end{aligned}
$$

where $\partial_{s}^{-1}:[n-1] \rightarrow[n] \backslash\{s\}$ is the inverse map of $\partial_{s}$.

Lemma 5.1. Suppose that $\mathcal{C}$ is $\mathrm{FI}_{G}$.

(i) Let $\alpha=(f, c) \in \mathfrak{C}(n, l)$ and $\beta \in \mathfrak{C}(m, n)$. Then

$$
\alpha \Psi_{n, 0}(\beta)=\Psi_{l, 0}(\alpha \beta)+\sum_{r \in[l] \backslash \operatorname{Im}_{g \in G}(f)} \Psi_{l, r, g}\left(\partial_{r} \alpha \beta\right) .
$$

(ii) Let $\alpha=(f, c) \in \mathfrak{C}(n, l), \beta \in \mathfrak{C}(m, n-1), s \in[n]$, and $h \in G$. Then

$$
\alpha \Psi_{n, s, h}(\beta)=\Psi_{l, f(s), h \cdot c(s)^{-1}}\left(\alpha_{s} \beta\right) .
$$

Proof. (i) Suppose $\gamma \in \mathfrak{C}(l, i)$. Then

$$
\begin{aligned}
\alpha \Psi_{n, 0}(\beta)\left(\Phi_{l, 0}(\gamma)\right) & =\Psi_{n, 0}(\beta)\left(\iota(\gamma) \circ\left(f_{l}, c_{l}\right) \circ \alpha\right) \\
& =\Psi_{n, 0}(\beta)\left(\iota(\gamma) \circ \iota(\alpha) \circ\left(f_{n}, c_{n}\right)\right) \\
& =\Psi_{n, 0}(\beta)\left(\Phi_{n, 0}(\gamma \alpha)\right) \\
& =\gamma \alpha \beta \\
& =\Psi_{l, 0}(\alpha \beta)\left(\Phi_{l, 0}(\gamma)\right) .
\end{aligned}
$$


Suppose $\gamma \in \mathcal{C}(l-1, i), r \in[l] \backslash \operatorname{Im}(f)$, and $g \in G$. Then

$$
\begin{aligned}
\alpha \Psi_{n, 0}(\beta)\left(\Phi_{l, r, g}(\gamma)\right) & =\Psi_{n, 0}(\beta)\left(\iota(\gamma) \circ\left(f_{l, r, g}, c_{l, r, g}\right) \circ \alpha\right) \\
& =\Psi_{n, 0}(\beta)\left(\iota(\gamma) \circ \iota\left(\partial_{r} \alpha\right) \circ\left(f_{n}, c_{n}\right)\right) \\
& =\Psi_{n, 0}(\beta)\left(\Phi_{n, 0}\left(\gamma \partial_{r} \alpha\right)\right) \\
& =\gamma \partial_{r} \alpha \beta \\
& =\Psi_{l, r, g}\left(\partial_{r} \alpha \beta\right)\left(\Phi_{l, r, g}(\gamma)\right) .
\end{aligned}
$$

Suppose $\gamma \in \mathcal{C}(l-1, i), r \in \operatorname{Im}(f)$, and $g \in G$. Then

$$
\begin{aligned}
\alpha \Psi_{n, 0}(\beta)\left(\Phi_{l, r, g}(\gamma)\right) & =\Psi_{n, 0}(\beta)\left(\iota(\gamma) \circ\left(f_{l, r, g}, c_{l, r, g}\right) \circ \alpha\right) \\
& =0 .
\end{aligned}
$$

(ii) Suppose $\gamma \in \mathcal{C}(l, i)$. Then

$$
\begin{aligned}
\alpha \Psi_{n, s, h}(\beta)\left(\Phi_{l, 0}(\gamma)\right) & =\Psi_{n, s, h}(\beta)\left(\iota(\gamma) \circ\left(f_{l}, c_{l}\right) \circ \alpha\right) \\
& =\Psi_{n, s, h}(\beta)\left(\iota(\gamma) \circ \iota(\alpha) \circ\left(f_{n}, c_{n}\right)\right) \\
& =0 .
\end{aligned}
$$

Suppose $\gamma \in \mathcal{C}(l-1, i), r \in[l]$, and $g \in G$.

If $r=f(s)$ and $g \cdot c(s)=h$, then

$$
\begin{aligned}
\alpha \Psi_{n, s, h}(\beta)\left(\Phi_{l, r, g}(\gamma)\right) & =\Psi_{n, s, h}(\beta)\left(\iota(\gamma) \circ\left(f_{l, r, g}, c_{l, r, g}\right) \circ \alpha\right) \\
& =\Psi_{n, s, h}(\beta)\left(\iota(\gamma) \circ \iota\left(\alpha_{s}\right) \circ\left(f_{n, s, h}, c_{n, s, h}\right)\right) \\
& =\Psi_{n, s, h}(\beta)\left(\Phi_{n, s, h}\left(\gamma \alpha_{s}\right)\right) \\
& =\gamma \alpha_{s} \beta \\
& =\Psi_{l, f(s), h \cdot c(s)^{-1}}\left(\alpha_{s} \beta\right)\left(\Phi_{l, r, g}(\gamma)\right)
\end{aligned}
$$

If $r \neq f(s)$ or $g \cdot c(s) \neq h$, then

$$
\begin{aligned}
\alpha \Psi_{n, s, h}(\beta)\left(\Phi_{l, r, g}(\gamma)\right) & =\Psi_{n, s, h}(\beta)\left(\iota(\gamma) \circ\left(f_{l, r, g}, c_{l, r, g}\right) \circ \alpha\right) \\
& =0 .
\end{aligned}
$$

5.3. Proof of Theorem 1.3, We retain the notations of subsection 5.2 ,

Proof of Theorem 1.3, Let $\mathrm{C}$ be $\mathrm{FI}_{G}$. Let $m \in \mathrm{Ob}(\mathrm{C})$. We need to prove that $\mathrm{Q}\left(k \mathrm{C} e_{m}\right)$ is isomorphic to $k \mathrm{C} e_{m} \oplus k \mathrm{C} e_{m+1}$.

For each $n \in \mathrm{Ob}(\mathcal{C})$, let $U(n)$ be the image of $\bigoplus_{r \in[n], g \in G} k \mathcal{C}(m, n-1)$ in $\mathrm{Q}\left(k \mathcal{C} e_{m}\right)(n)$ under $\Psi_{n}$, and let $U=\bigoplus_{n \in \mathrm{Ob}(\mathcal{C})} U(n)$. By Lemma 5.1, $U$ is a $\mathcal{C}$-submodule of $\mathrm{Q}\left(k \mathrm{C} e_{m}\right)$ and there is a short exact sequence

$$
0 \longrightarrow U \longrightarrow \mathrm{Q}\left(k \mathrm{C} e_{m}\right) \longrightarrow k \mathrm{C} e_{m} \longrightarrow 0 .
$$

Since $k \mathcal{C} e_{m}$ is projective, this short exact sequence splits. It suffices to show that $U$ is isomorphic to $k \mathrm{C} e_{m+1}$. 
For each $n \in \mathrm{Ob}(\mathcal{C})$, define a linear map $\Theta_{n}: U(n) \rightarrow k \mathcal{C}(m+1, n)$ by

$$
\Theta_{n}\left(\Psi_{n, s, h}(\beta)\right)=\left(f_{n, s, h}, c_{n, s, h}\right)^{-1} \iota(\beta)
$$

for all $s \in[n], h \in G$, and $\beta \in \mathcal{C}(m, n-1)$. Let $\Theta: U \rightarrow k \mathfrak{C} e_{m+1}$ be the linear map whose restriction to $U(n)$ is $\Theta_{n}$. It is easy to see that $\Theta$ is bijective.

We show now that $\Theta$ is a $\mathcal{C}$-module homomorphism. Suppose $\alpha=(f, c) \in \mathcal{C}(n, l), \beta \in$ $\mathcal{C}(m, n-1), s \in[n]$, and $h \in G$. Observe that

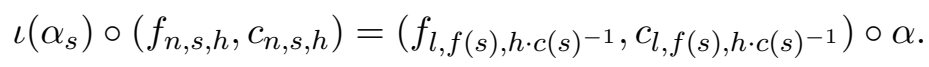

Hence,

$$
\begin{aligned}
\Theta_{l}\left(\alpha \Psi_{n, s, h}(\beta)\right) & =\Theta_{l}\left(\Psi_{l, f(s), h \cdot c(s)^{-1}}\left(\alpha_{s} \beta\right)\right) \\
& =\left(f_{\left.l, f(s), h \cdot c(s)^{-1}, c_{l, f(s), h \cdot c(s)^{-1}}\right)^{-1} \iota\left(\alpha_{s} \beta\right)}\right. \\
& =\alpha\left(f_{n, s, h}, c_{n, s, h}\right)^{-1} \iota(\beta) \\
& =\alpha \Theta_{n}\left(\Psi_{n, s, h}(\beta)\right) .
\end{aligned}
$$

\section{Structure of $\mathrm{Q}\left(k \mathcal{C} e_{m}\right)$ when $\mathcal{C}$ is VI}

Throughout this section, $k$ denotes any commutative ring.

6.1. Structure of $\mathrm{S}\left(k \mathrm{C} e_{n}\right)$. Suppose that $\mathcal{C}$ is VI. Let $n \in \mathrm{Ob}(\mathcal{C})$. We now recall the structure of $\mathrm{S}\left(k \mathcal{C} e_{n}\right)$.

We write elements $\alpha \in \mathcal{C}(n, l)$ as a $l \times n$-matrix. We write elements $u \in \mathbb{F}^{n}$ as a column vector and $u^{t}$ for its transpose. Let $\mathrm{P}\left(\mathbb{F}^{n}\right)$ be the set of one dimensional vector subspaces of $\mathbb{F}^{n}$. For any $u \in \mathbb{F}^{n}$ and $\ell \in \mathrm{P}\left(\mathbb{F}^{n}\right)$, we write $u^{t}(\ell) \neq 0$ if $u^{t} v \neq 0$ for any nonzero vector $v$ in $\ell$. For each $\ell \in \mathrm{P}\left(\mathbb{F}^{n}\right)$, we choose and fix a $(n-1) \times n$-matrix $\varpi_{\ell}: \mathbb{F}^{n} \rightarrow \mathbb{F}^{n-1}$ whose kernel is $\ell$. We shall denote identity matrices by $I$.

Now, for any $u \in \mathbb{F}^{n}$ and $\ell \in \mathrm{P}\left(\mathbb{F}^{n}\right)$ such that $u^{t}(\ell) \neq 0$, define the maps

$$
\begin{gathered}
\Phi_{n, u, 0}: \mathcal{C}(n, l) \longrightarrow \mathcal{C}(n, l+1), \quad \alpha \mapsto\left(\begin{array}{ll}
1 & 0 \\
0 & \alpha
\end{array}\right)\left(\begin{array}{c}
u^{t} \\
I
\end{array}\right) ; \\
\Phi_{n, u, \ell}: \mathcal{C}(n-1, l) \longrightarrow \mathcal{C}(n, l+1), \quad \alpha \mapsto\left(\begin{array}{ll}
1 & 0 \\
0 & \alpha
\end{array}\right)\left(\begin{array}{c}
u^{t} \\
\varpi_{\ell}
\end{array}\right) .
\end{gathered}
$$

We may extend these maps linearly to $\mathcal{C}$-module homomorphisms

$$
\Phi_{n, u, 0}: k \mathcal{C} e_{n} \longrightarrow \mathrm{S}\left(k \mathcal{C} e_{n}\right) \quad \text { and } \quad \Phi_{n, u, \ell}: k \mathcal{C} e_{n-1} \longrightarrow \mathrm{S}\left(k \mathcal{C} e_{n}\right),
$$

Let

$$
\Phi_{n}:\left(\bigoplus_{u \in \mathbb{F}^{n}} k \mathcal{C} e_{n}\right) \oplus\left(\bigoplus_{\substack{u \in \mathbb{F}^{n} \\ \ell \in \mathrm{P}\left(\mathbb{F}^{n}\right) \\ u^{t}(\ell) \neq 0}} k \mathcal{C} e_{n-1}\right) \longrightarrow \mathrm{S}\left(k \mathcal{C} e_{n}\right)
$$

be the $\mathcal{C}$-module homomorphism whose restriction to the direct summand $k \mathcal{C} e_{n}$ indexed by $u \in \mathbb{F}^{n}$ is $\Phi_{n, u, 0}$ and whose restriction to the direct summand $k \bigodot e_{n-1}$ indexed by $u \in \mathbb{F}^{n}$, 
$\ell \in \mathrm{P}\left(\mathbb{F}^{n}\right)$ is $\Phi_{n, u, \ell}$. It is straightforward to verify that $\Phi_{n}$ is an isomorphism (see [8, Section $5])$. Thus, $\iota$ is a genetic functor.

6.2. Preliminary discussion of $\mathrm{Q}\left(k \bigodot e_{m}\right)$. We retain the notations of subsection 6.1, Let $m, n \in \mathrm{Ob}(\mathcal{C})$. By the isomorphism (6.1), one has the identification

$$
\mathrm{Q}\left(k \mathcal{C} e_{m}\right)(n)=\left(\bigoplus_{u \in \mathbb{F}^{n}} \operatorname{Hom}_{\mathcal{C}}\left(k \mathcal{C} e_{n}, k \mathcal{C} e_{m}\right)\right) \oplus\left(\bigoplus_{\substack{u \in \mathbb{F}^{n} \\ \ell \in \mathrm{P}\left(\mathbb{F}^{n}\right) \\ u^{t}(\ell) \neq 0}} \operatorname{Hom}_{\mathcal{C}}\left(k \mathcal{C} e_{n-1}, k \mathcal{C} e_{m}\right)\right)
$$

Denote by

$$
\begin{aligned}
\Psi_{n, u, 0}: k \mathrm{C}(m, n) & \longrightarrow \mathrm{Q}\left(k \mathrm{C} e_{m}\right)(n), \\
\Psi_{n, u, \ell}: k \mathrm{C}(m, n-1) & \longrightarrow \mathrm{Q}\left(k \mathcal{C} e_{m}\right)(n)
\end{aligned}
$$

the linear maps where $\Psi_{n, u, 0}$ is the natural bijection of $k \mathrm{C}(m, n)$ with the direct summand $\operatorname{Hom}_{\mathcal{C}}\left(k \mathrm{C} e_{n}, k \mathcal{C} e_{m}\right)$ of $\mathrm{Q}\left(k \mathcal{C} e_{m}\right)(n)$ indexed by $u \in \mathbb{F}^{n}$, and $\Psi_{n, u, \ell}$ is the natural bijection of $k \mathrm{C}(m, n-1)$ with the direct summand $\operatorname{Hom}_{\mathcal{C}}\left(k \mathrm{C} e_{n-1}, k \mathrm{C} e_{m}\right)$ of $\mathrm{Q}\left(k \mathcal{C} e_{m}\right)(n)$ indexed by $u \in \mathbb{F}^{n}, \ell \in \mathrm{P}\left(\mathbb{F}^{n}\right)$. We have a linear bijection

$$
\Psi_{n}:\left(\bigoplus_{u \in \mathbb{F}^{n}} k \mathrm{C}(m, n)\right) \oplus\left(\bigoplus_{\substack { u \in \mathbb{F}^{n} \\
\begin{subarray}{c}{\ell \in \mathrm{P}\left(\mathbb{F}^{n}\right) \\
u^{t}(\ell) \neq 0{ u \in \mathbb { F } ^ { n } \\
\begin{subarray} { c } { \ell \in \mathrm { P } ( \mathbb { F } ^ { n } ) \\
u ^ { t } ( \ell ) \neq 0 } }\end{subarray}} k \mathrm{C}(m, n-1)\right) \rightarrow \mathrm{Q}\left(k \mathrm{C} e_{m}\right)(n)
$$

whose restriction to the direct summand $k \mathrm{C}(m, n)$ indexed by $u \in \mathbb{F}^{n}$ is $\Psi_{n, u, 0}$ and whose restriction to the direct summand $k \mathrm{C}(m, n-1)$ indexed by $u \in \mathbb{F}^{n}, \ell \in \mathrm{P}\left(\mathbb{F}^{n}\right)$ is $\Psi_{n, u, \ell}$.

The next lemma describes the $\mathcal{C}$-module structure of $\mathrm{Q}\left(k \mathcal{C} e_{m}\right)$ in terms of the identifications $\Psi_{n}$ for $n \in \mathrm{Ob}(\mathcal{C})$. We shall use the following notation. For any $\alpha \in \mathcal{C}(n, l)$ and $\wp \in \mathrm{P}\left(\mathbb{F}^{n}\right)$, let $\alpha_{\wp} \in \mathcal{C}(n-1, l-1)$ be the unique linear map such that the following diagram commutes:

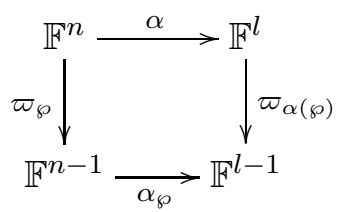

Lemma 6.1. Suppose that $\mathcal{C}$ is VI.

(i) Let $\alpha \in \mathfrak{C}(n, l), \beta \in \mathfrak{C}(m, n)$, and $v \in \mathbb{F}^{n}$. Then

$$
\alpha \Psi_{n, v, 0}(\beta)=\sum_{\substack{u \in \mathbb{F}^{l} \\ u^{t} \alpha=v^{t}}} \Psi_{l, u, 0}(\alpha \beta)+\sum_{\substack{u \in \mathbb{F}^{l} \\ u^{t} \alpha=v^{t}}} \sum_{\substack{\ell \in \mathrm{P}\left(\mathbb{F}^{l}\right) \\ u^{t}(\ell) \neq 0 \\ \ell \nsubseteq \operatorname{Im}(\alpha)}} \Psi_{l, u, \ell}\left(\varpi_{\ell} \alpha \beta\right) .
$$

(ii) Let $\alpha \in \mathfrak{C}(n, l), \beta \in \mathfrak{C}(m, n-1), v \in \mathbb{F}^{n}$, and $\wp \in \mathrm{P}\left(\mathbb{F}^{n}\right)$. Suppose $v^{t}(\wp) \neq 0$. Then

$$
\alpha \Psi_{n, v, \wp}(\beta)=\sum_{\substack{u \in \mathbb{F}^{l} \\ u^{t} \alpha=v^{t}}} \sum_{\substack{\ell \in \mathrm{P}\left(\mathbb{F}^{l}\right) \\ \ell=\alpha(\wp)}} \Psi_{l, u, \ell}\left(\alpha_{\wp} \beta\right) .
$$


Proof. (i) Suppose $\gamma \in \mathcal{C}(l, i)$ and $u \in \mathbb{F}^{l}$. One has

$$
\Phi_{l, u, 0}(\gamma) \alpha=\left(\begin{array}{cc}
1 & 0 \\
0 & \gamma
\end{array}\right)\left(\begin{array}{c}
u^{t} \alpha \\
\alpha
\end{array}\right)=\left(\begin{array}{cc}
1 & 0 \\
0 & \gamma \alpha
\end{array}\right)\left(\begin{array}{c}
u^{t} \alpha \\
I
\end{array}\right)
$$

Thus,

$$
\alpha \Psi_{n, v, 0}(\beta)\left(\Phi_{l, u, 0}(\gamma)\right)=\Psi_{n, v, 0}(\beta)\left(\Phi_{l, u, 0}(\gamma) \alpha\right)= \begin{cases}0 & \text { if } u^{t} \alpha \neq v^{t} \\ \gamma \alpha \beta & \text { if } u^{t} \alpha=v^{t}\end{cases}
$$

In particular, when $u^{t} \alpha=v^{t}$, one has

$$
\alpha \Psi_{n, v, 0}(\beta)\left(\Phi_{l, u, 0}(\gamma)\right)=\Psi_{l, u, 0}(\alpha \beta)\left(\Phi_{l, u, 0}(\gamma)\right) .
$$

Now suppose $\gamma \in \mathcal{C}(l-1, i), u \in \mathbb{F}^{l}, \ell \in \mathrm{P}\left(\mathbb{F}^{l}\right)$, and $u^{t}(\ell) \neq 0$. One has

$$
\Phi_{l, u, \ell}(\gamma) \alpha=\left(\begin{array}{cc}
1 & 0 \\
0 & \gamma
\end{array}\right)\left(\begin{array}{c}
u^{t} \alpha \\
\varpi_{\ell} \alpha
\end{array}\right)=\left(\begin{array}{cc}
1 & 0 \\
0 & \gamma \varpi_{\ell} \alpha
\end{array}\right)\left(\begin{array}{c}
u^{t} \alpha \\
I
\end{array}\right)
$$

Thus,

$$
\begin{aligned}
\alpha \Psi_{n, v, 0}(\beta)\left(\Phi_{l, u, \ell}(\gamma)\right) & =\Psi_{n, v, 0}(\beta)\left(\Phi_{l, u, \ell}(\gamma) \alpha\right) \\
& = \begin{cases}0 & \text { if } u^{t} \alpha \neq v^{t} \text { or } \ell \subseteq \operatorname{Im}(\alpha), \\
\gamma \varpi_{\ell} \alpha \beta & \text { if } u^{t} \alpha=v^{t} \text { and } \ell \nsubseteq \operatorname{Im}(\alpha) .\end{cases}
\end{aligned}
$$

In particular, when $u^{t} \alpha=v^{t}$ and $\ell \nsubseteq \operatorname{Im}(\alpha)$, one has

$$
\alpha \Psi_{n, v, 0}(\beta)\left(\Phi_{l, u, \ell}(\gamma)\right)=\Psi_{l, u, \ell}\left(\varpi_{\ell} \alpha \beta\right)\left(\Phi_{l, u, \ell}(\gamma)\right) .
$$

(ii) Suppose $\gamma \in \mathcal{C}(l, i)$ and $u \in \mathbb{F}^{l}$. From (6.2), one has

$$
\alpha \Psi_{n, v, \wp}(\beta)\left(\Phi_{l, u, 0}(\gamma)\right)=\Psi_{n, v, \wp}(\beta)\left(\Phi_{l, u, 0}(\gamma) \alpha\right)=0 .
$$

Now suppose $\gamma \in \mathcal{C}(l-1, i), u \in \mathbb{F}^{l}, \ell \in \mathrm{P}\left(\mathbb{F}^{l}\right)$, and $u^{t}(\ell) \neq 0$. One has

$$
\Phi_{l, u, \ell}(\gamma) \alpha=\left(\begin{array}{cc}
1 & 0 \\
0 & \gamma
\end{array}\right)\left(\begin{array}{c}
u^{t} \alpha \\
\varpi_{\ell} \alpha
\end{array}\right)
$$

We can write $\left(\begin{array}{c}u^{t} \alpha \\ \varpi_{\ell} \alpha\end{array}\right)$ in the form $\left(\begin{array}{ll}1 & 0 \\ 0 & *\end{array}\right)\left(\begin{array}{c}u^{t} \alpha \\ \varpi_{\wp}\end{array}\right)$ if and only if $\ell=\alpha(\wp)$. If $\ell=\alpha(\wp)$, then

$$
\Phi_{l, u, \ell}(\gamma) \alpha=\left(\begin{array}{cc}
1 & 0 \\
0 & \gamma \alpha_{\wp}
\end{array}\right)\left(\begin{array}{l}
u^{t} \alpha \\
\varpi_{\wp}
\end{array}\right) \text {. }
$$

Thus,

$$
\begin{aligned}
\alpha \Psi_{n, v, \wp}(\beta) \Phi_{l, u, \ell}(\gamma) & =\Psi_{n, v, \wp}(\beta)\left(\Phi_{l, u, \ell}(\gamma) \alpha\right) \\
& = \begin{cases}0 & \text { if } u^{t} \alpha \neq v^{t} \text { or } \ell \neq \alpha(\wp), \\
\gamma \alpha_{\wp} \beta & \text { if } u^{t} \alpha=v^{t} \text { and } \ell=\alpha(\wp) .\end{cases}
\end{aligned}
$$

In particular, when $u^{t} \alpha=v^{t}$ and $\ell=\alpha(\wp)$, one has

$$
\alpha \Psi_{n, v, \wp}(\beta)\left(\Phi_{l, u, \ell}(\gamma)\right)=\Psi_{l, u, \ell}\left(\alpha_{\wp} \beta\right)\left(\Phi_{l, u, \ell}(\gamma)\right) .
$$


6.3. Proof of Theorem 1.4, We retain the notations of subsection 6.2.

Proof of Theorem 1.4. Let $\mathcal{C}$ be VI. Let $m \in \mathrm{Ob}(\mathcal{C})$. We need to prove that $\mathrm{Q}\left(k \mathcal{C} e_{m}\right)$ contains a direct summand isomorphic to $k \mathcal{C} e_{m+1}$.

Since $k \mathcal{C} e_{m+1}$ is a projective $\mathcal{C}$-module, it suffices to construct a surjective homomorphism

$$
\pi: \mathrm{Q}\left(k \mathrm{C} e_{m}\right) \longrightarrow k \mathrm{C} e_{m+1}
$$

For each $n \in \mathrm{Ob}(\mathcal{C})$, we define a linear map $\pi_{n}: \mathrm{Q}\left(k \mathcal{C} e_{m}\right)(n) \longrightarrow k \mathcal{C}(m+1, n)$ as follows:

(1) If $\beta \in \mathcal{C}(m, n)$ and $v \in \mathbb{F}^{n}$, let

$$
\pi_{n}\left(\Psi_{n, v, 0}(\beta)\right)=-q^{-n} \sum_{\substack{\wp \in \mathrm{P}\left(\mathbb{F}^{n}\right) \\
v^{t}(\wp) \neq 0 \\
\wp \nsubseteq \operatorname{Im}(\beta)}}\left(\begin{array}{c}
v^{t} \\
\varpi_{\wp}
\end{array}\right)^{-1}\left(\begin{array}{cc}
1 & 0 \\
0 & \varpi_{\wp} \beta
\end{array}\right) .
$$

(2) If $\beta \in \mathcal{C}(m, n-1), v \in \mathbb{F}^{n}, \wp \in \mathrm{P}\left(\mathbb{F}^{n}\right)$, and $v^{t}(\wp) \neq 0$, let

$$
\pi_{n}\left(\Psi_{n, v, \wp}(\beta)\right)=q^{-n}\left(\begin{array}{c}
v^{t} \\
\varpi_{\wp}
\end{array}\right)^{-1}\left(\begin{array}{cc}
1 & 0 \\
0 & \beta
\end{array}\right) .
$$

Let $\pi$ be the linear map whose restriction to $\mathrm{Q}\left(k \mathcal{C} e_{m}\right)(n)$ is $\pi_{n}$. We claim that: $\pi$ is surjective, and $\pi$ is a $\mathcal{C}$-module homomorphism.

To show that $\pi$ is surjective, consider any $\gamma \in \mathcal{C}(m+1, n)$. We want to show that $\gamma$ is in the image of $\pi_{n}$. To this end, we write the $m+1$ columns of $\gamma$ as $\gamma_{1}, \ldots, \gamma_{m+1} \in \mathbb{F}^{n}$. Let $\wp \in \mathrm{P}\left(\mathbb{F}^{n}\right)$ be the span of $\gamma_{1}$; so one has $\varpi_{\wp} \gamma_{1}=0$. Since $\gamma: \mathbb{F}^{m+1} \rightarrow \mathbb{F}^{n}$ is injective, its transpose $\gamma^{t}: \mathbb{F}^{n} \rightarrow \mathbb{F}^{m+1}$ is surjective. Hence, there exists $v \in \mathbb{F}^{n}$ such that

$$
v^{t} \gamma_{1}=1, \quad v^{t} \gamma_{2}=\cdots v^{t} \gamma_{m+1}=0 .
$$

Choose such a $v$. Then one has

$$
\left(\begin{array}{c}
v^{t} \\
\varpi_{\wp}
\end{array}\right) \gamma=\left(\begin{array}{ll}
1 & 0 \\
0 & \beta
\end{array}\right)
$$

for some $\beta \in \mathfrak{C}(m, n-1)$. Therefore,

$$
\pi_{n}\left(q^{n} \Psi_{n, v, \wp}(\beta)\right)=\left(\begin{array}{c}
v^{t} \\
\varpi_{\wp}
\end{array}\right)^{-1}\left(\begin{array}{cc}
1 & 0 \\
0 & \beta
\end{array}\right)=\gamma .
$$

It remains to check that $\pi$ is a $\mathcal{C}$-module homomorphism. Let $\alpha \in \mathfrak{C}(n, l)$.

Observe that if $v \in \mathbb{F}^{n}$, then

$$
\#\left\{u \in \mathbb{F}^{l} \mid u^{t} \alpha=v^{t}\right\}=q^{l-n} .
$$

Suppose that $\beta \in \mathfrak{C}(m, n)$ and $v \in \mathbb{F}^{n}$. One has: 


$$
\begin{aligned}
& \pi_{l}\left(\alpha \Psi_{n, v, 0}(\beta)\right) \\
& =\sum_{\substack{u \in \mathbb{F}^{l} \\
u^{t} \alpha=v^{t}}} \pi_{l}\left(\Psi_{l, u, 0}(\alpha \beta)\right)+\sum_{\substack{u \in \mathbb{F}^{l} \\
u^{t} \alpha=v^{t}}} \sum_{\substack{\ell \in \mathrm{P}\left(\mathbb{F}^{l}\right) \\
u^{t}(\ell) \neq 0}} \pi_{l}\left(\Psi_{l, u, \ell}\left(\varpi_{\ell} \alpha \beta\right)\right) \\
& =-q^{-l} \sum_{\substack{u \in \mathbb{F}^{l} \\
u^{t} \alpha=v^{t}}} \sum_{\substack{\ell \in \mathrm{P}\left(\mathbb{F}^{l}\right) \\
u^{t}(\ell) \neq 0 \\
\ell \nsubseteq \operatorname{Im}(\alpha \beta)}}\left(\begin{array}{c}
u^{t} \\
\varpi_{\ell}
\end{array}\right)^{-1}\left(\begin{array}{cc}
1 & 0 \\
0 & \varpi_{\ell} \alpha \beta
\end{array}\right) \\
& +q^{-l} \sum_{\substack{u \in \mathbb{F}^{l} \\
u^{t} \alpha=v^{t}}} \sum_{\substack{\ell \in \mathrm{P}\left(\mathbb{F}^{l}\right) \\
u^{t}(\ell) \neq 0 \\
\ell \nsubseteq \operatorname{Im}(\alpha)}}\left(\begin{array}{c}
u^{t} \\
\varpi_{\ell}
\end{array}\right)^{-1}\left(\begin{array}{cc}
1 & 0 \\
0 & \varpi_{\ell} \alpha \beta
\end{array}\right) \\
& =-q^{-l} \sum_{\substack{u \in \mathbb{F}^{l} \\
u^{t} \alpha=v^{t}}} \sum_{\substack{\ell \in \mathrm{P}\left(\mathbb{F}^{l}\right) \\
u^{t}(\ell) \neq 0 \\
\ell \subseteq \operatorname{Im}(\alpha) \backslash \operatorname{Im}(\alpha \beta)}}\left(\begin{array}{c}
u^{t} \\
\varpi_{\ell}
\end{array}\right)^{-1}\left(\begin{array}{cc}
1 & 0 \\
0 & \varpi_{\ell} \alpha \beta
\end{array}\right) \\
& =-q^{-l} \sum_{\substack{u \in \mathbb{F}^{l} \\
u^{t} \alpha=v^{t}}} \sum_{\substack{\wp \in \mathrm{P}\left(\mathbb{F}^{n}\right) \\
v^{t}(\wp) \neq 0 \\
\wp \nsubseteq \operatorname{Im}(\beta)}}\left(\begin{array}{c}
u^{t} \\
\varpi_{\alpha(\wp)}
\end{array}\right)^{-1}\left(\begin{array}{cc}
1 & 0 \\
0 & \varpi_{\alpha(\wp)} \alpha \beta
\end{array}\right) \\
& =-q^{-l} \sum_{\substack{u \in \mathbb{F}^{l} \\
u^{t} \alpha=v^{t}}} \sum_{\substack{\wp \in \mathrm{P}\left(\mathbb{F}^{n}\right) \\
v^{t}(\wp) \neq 0 \\
\wp \nsubseteq \operatorname{Im}(\beta)}}\left(\begin{array}{c}
u^{t} \\
\varpi_{\alpha(\wp)}
\end{array}\right)^{-1}\left(\begin{array}{cc}
1 & 0 \\
0 & \alpha_{\wp} \varpi_{\wp} \beta
\end{array}\right) \text {. }
\end{aligned}
$$

Observe that when $u^{t} \alpha=v^{t}$, one has

$$
\left(\begin{array}{c}
u^{t} \\
\varpi_{\alpha(\wp)}
\end{array}\right) \alpha=\left(\begin{array}{c}
v^{t} \\
\alpha_{\wp} \varpi_{\wp}
\end{array}\right)=\left(\begin{array}{cc}
1 & 0 \\
0 & \alpha_{\wp}
\end{array}\right)\left(\begin{array}{c}
v^{t} \\
\varpi_{\wp}
\end{array}\right)
$$

which implies

$$
\left(\begin{array}{c}
u^{t} \\
\varpi_{\alpha(\wp)}
\end{array}\right)^{-1}\left(\begin{array}{cc}
1 & 0 \\
0 & \alpha_{\wp}
\end{array}\right)=\alpha\left(\begin{array}{c}
v^{t} \\
\varpi_{\wp}
\end{array}\right)^{-1} .
$$


Hence, continuing our calculation from above,

$$
\begin{aligned}
& \pi_{l}\left(\alpha \Psi_{n, v, 0}(\beta)\right) \\
= & -q^{-l} \sum_{\substack{u \in \mathbb{F}^{l} \\
u^{t} \alpha=v^{t}}} \sum_{\substack{\wp \in \mathrm{P}\left(\mathbb{F}^{n}\right) \\
v^{t}(\wp) \neq 0 \\
\wp \nsubseteq \operatorname{Im}(\beta)}} \alpha\left(\begin{array}{c}
v^{t} \\
\varpi_{\wp}
\end{array}\right)^{-1}\left(\begin{array}{cc}
1 & 0 \\
0 & \varpi_{\wp} \beta
\end{array}\right) \\
= & -q^{-l} \cdot q^{l-n} \sum_{\substack{\wp \in \mathrm{P}\left(\mathbb{F}^{n}\right) \\
v^{t}(\wp) \neq 0 \\
\wp \nsubseteq \operatorname{Im}(\beta)}} \alpha\left(\begin{array}{c}
v^{t} \\
\varpi_{\wp}
\end{array}\right)^{-1}\left(\begin{array}{cc}
1 & 0 \\
0 & \varpi_{\wp} \beta
\end{array}\right) \\
= & \alpha \pi_{n}\left(\Psi_{n, v, 0}(\beta)\right) .
\end{aligned}
$$

Now suppose that $\beta \in \mathcal{C}(m, n-1), v \in \mathbb{F}^{n}, \wp \in \mathrm{P}\left(\mathbb{F}^{n}\right)$, and $v^{t}(\wp) \neq 0$. One has:

$$
\begin{aligned}
& \sum_{l}\left(\alpha \Psi_{n, v, \wp}(\beta)\right) \\
= & \sum_{\substack{u \in \mathbb{F}^{l} \\
u^{t} \alpha=v^{t}}} \pi_{\ell=\alpha(\wp)}\left(\Psi_{l, u, \ell}\left(\alpha_{\wp} \beta\right)\right) \\
= & q^{-l} \sum_{\substack{u \in \mathbb{F}^{l} \\
u^{t} \alpha=v^{t}}} \sum_{\substack{\ell \in \mathrm{P}\left(\mathbb{F}^{l}\right) \\
\ell=\alpha(\wp)}}\left(\begin{array}{c}
u^{t} \\
\varpi_{\ell}
\end{array}\right)^{-1}\left(\begin{array}{cc}
1 & 0 \\
0 & \alpha_{\wp} \beta
\end{array}\right) \\
= & q^{-l} \sum_{\substack{u \in \mathbb{F}^{l} \\
u^{t} \alpha=v^{t}}} \sum_{\ell=\mathrm{P}\left(\mathbb{F}^{l}\right)} \alpha\left(\begin{array}{c}
v^{t} \\
\varpi_{\wp}
\end{array}\right)^{-1}\left(\begin{array}{cc}
1 & 0 \\
0 & \beta
\end{array}\right) \quad \text { using (6.3) } \\
= & q^{-l} \cdot q^{l-n} \cdot \alpha\left(\begin{array}{c}
v^{t} \\
\varpi_{\wp}
\end{array}\right)^{-1}\left(\begin{array}{cc}
1 & 0 \\
0 & \beta
\end{array}\right) \\
= & \alpha \pi_{n}\left(\Psi_{n, v, \wp}(\beta)\right) .
\end{aligned}
$$

This completes the verification that $\pi$ is a $\mathcal{C}$-module homomorphism.

\section{Applications of Coinduction functor}

Throughout this section, we assume that $k$ is a field of characteristic 0 .

7.1. Proofs of Theorem 1.5 and Corollary 1.6. Suppose that $\mathcal{C}$ is $\mathrm{FI}_{G}$ or VI. Recall that $\mathcal{C}$ is locally Noetherian by [7].

Proof of Theorem 1.5. It suffices to prove that $k e_{n}$ is injective for each $n \in \mathrm{Ob}(\mathcal{C})$. We prove this by induction on $n$.

By Corollary [3.2, $k \mathrm{C} e_{n}$ is injective when $n=0$. Now suppose that $k \mathrm{C} e_{n}$ is injective when $n=m$ for some $m \in \mathrm{Ob}(\mathcal{C})$. By Lemma 4.3, $\mathrm{Q}\left(k \mathcal{C} e_{m}\right)$ is injective. But $k \mathcal{C} e_{m+1}$ is a direct summand of $\mathrm{Q}\left(k \bigodot e_{m}\right)$ by Theorems 1.3 and 1.4. It follows that $k \bigodot e_{n}$ is injective for $n=m+1$. 
To carry out the inductive argument in the above proof, we need to know that $\mathrm{Q}\left(k \mathrm{C} e_{m}\right)$ contains a direct summand isomorphic to $k \mathrm{Ce}_{m+1}$. This was verified for the categories $\mathrm{FI}_{G}$ and VI by the explicit computations in Sections 5 and 6, we do not know if a conceptual proof can be given based on certain underlying combinatorial properties of the category $\mathcal{C}$.

Proof of Corollary 1.6. Suppose $V$ is a finitely generated $\mathcal{C}$-module and

$$
0 \rightarrow P^{-r} \rightarrow \cdots \rightarrow P^{-1} \rightarrow P^{0} \rightarrow V \rightarrow 0
$$

is an exact sequence where $P^{0}, \ldots, P^{-r}$ are finitely generated projective $\mathcal{C}$-modules. Then there are short exact sequences $0 \rightarrow Q^{i-1} \rightarrow P^{i} \rightarrow Q^{i} \rightarrow 0$ for $i=0, \ldots,-(r-1)$ where $Q^{0}, \ldots, Q^{-r}$ are finitely generated $\mathcal{C}$-modules such that $Q^{0}=V$ and $Q^{-r}=P^{-r}$. It follows from Theorem 1.5 that these short exact sequences split; in particular, $V$ is a direct summand of $P^{0}$.

7.2. Torsion-free modules. Suppose that $\mathcal{C}$ is $\mathrm{FI}_{G}$.

Definition 7.1. A $\mathcal{C}$-module $F$ is torsion-free if $\operatorname{Hom}_{\mathcal{C}}(T, F)=0$ for all finite dimensional C-modules $T$.

Lemma 7.2. Suppose that $\mathcal{C}$ is $\mathrm{FI}_{G}$. Let $V$ be a finitely generated $\mathcal{C}$-module. Then there exists a short exact sequence

$$
0 \longrightarrow T \longrightarrow V \longrightarrow F \longrightarrow 0
$$

such that $T$ is a finite dimensional $\mathrm{C}$-module and $F$ is a torsion-free $\mathcal{C}$-module.

Proof. Since $V$ is Noetherian, there exists a maximal finite dimensional submodule $T$ of $V$. It is plain that $V / T$ is torsion-free.

Lemma 7.3. Suppose that $\mathcal{C}$ is $\mathrm{FI}_{G}$. Let $F$ be a finitely generated torsion-free $\mathcal{C}$-module. If $F \neq 0$, then there exists $n \in \mathrm{Ob}(\mathcal{C})$ such that $\operatorname{Hom}_{\mathfrak{C}}\left(F, k \mathcal{C} e_{n}\right) \neq 0$.

Proof. Since $F \neq 0$, there exists a smallest $a \in \mathrm{Ob}(\mathcal{C})$ such that $F(a) \neq 0$. Thus, $\mathrm{S}^{a}(F)(0) \neq$ 0 . Choose a nonzero element $s$ of $\mathrm{S}^{a}(F)(0)$ and let

$$
f: k \mathrm{C} e_{0} \longrightarrow \mathrm{S}^{a}(F), \quad \alpha \mapsto \alpha s .
$$

Since $F$ is torsion-free, the homomorphism $f$ is injective. But $k \mathcal{C}_{0}$ is an injective $\mathcal{C}$-module by Theorem 1.5. Thus, there exists a nonzero homomorphism from $\mathrm{S}^{a}(F)$ to $k \mathrm{C}_{0}$. It follows from Lemma 4.3 that

$$
\operatorname{Hom}_{\mathcal{C}}\left(F, \mathrm{Q}^{a}\left(k e_{0}\right)\right)=\operatorname{Hom}_{\mathcal{C}}\left(\mathrm{S}^{a}(F), k \bigodot e_{0}\right) \neq 0 .
$$

But by Theorem 1.3, $\mathrm{Q}^{a}\left(k \mathcal{C} e_{0}\right)$ is isomorphic to $k \mathcal{C} e_{n_{1}} \oplus \cdots \oplus k \mathcal{C} e_{n_{r}}$ for some $n_{1}, \ldots, n_{r} \in$ $\mathrm{Ob}(\mathcal{C})$. Hence, the result follows.

Suppose $V$ is a finitely generated $\mathcal{C}$-module. Then there exists $l \in \mathrm{Ob}(\mathcal{C})$ such that $V$ is generated in degrees $\leqslant l$; clearly, one has $\operatorname{Hom}_{\mathcal{C}}\left(V, k \mathcal{C} e_{n}\right)=0$ for all $n>l$.

Notation 7.4. For any finitely generated $\mathcal{C}$-module $V$, let

$$
\kappa(V)=\sum_{n \in \mathrm{Ob}(\mathcal{C})} \kappa(V, n)
$$

where $\kappa(V, n)=\operatorname{dim}_{k} \operatorname{Hom}_{\mathcal{C}}\left(V, k \mathcal{C} e_{n}\right)$ for each $n \in \mathrm{Ob}(\mathcal{C})$. 
Proposition 7.5. Suppose that $\mathcal{C}$ is $\mathrm{FI}_{G}$. Let $F$ be a finitely generated torsion-free $\mathcal{C}$ module. Then there exists an injective homomorphism from $F$ to $k \mathrm{C} e_{n_{1}} \oplus \cdots \oplus k \mathrm{C} e_{n_{r}}$ for some $n_{1}, \ldots, n_{r} \in \mathrm{Ob}(\mathcal{C})$.

Proof. If $\kappa(F)=0$, then $F=0$ by Lemma 7.3. We shall prove the proposition by induction on $\kappa(F)$. Suppose $\kappa(F)>0$. Then there exists a nonzero homomorphism $f: F \rightarrow k \mathcal{C} e_{m}$ for some $m \in \mathrm{Ob}(\mathcal{C})$. Let $E=\operatorname{Ker}(f)$ and $W=\operatorname{Im}(f)$. We have a short exact sequence

$$
0 \longrightarrow E \longrightarrow F \longrightarrow W \longrightarrow 0 \text {. }
$$

By Theorem 1.5, the $\mathcal{C}$-module $k \mathcal{C} e_{n}$ is injective, so

$$
\kappa(E, n)+\kappa(W, n)=\kappa(F, n) \quad \text { for each } n \in \mathrm{Ob}(\mathcal{C}) .
$$

Therefore, $\kappa(E)+\kappa(W)=\kappa(F)$. But $\kappa(W, m)>0$, so $\kappa(E)<\kappa(F)$. Observe that $E$ is a finitely generated torsion-free $\mathcal{C}$-module. By induction hypothesis, there exists an injective homomorphism from $E$ to $k \mathcal{C} e_{n_{1}} \oplus \cdots \oplus k \mathcal{C} e_{n_{r}}$ for some $n_{1}, \ldots, n_{r} \in \mathrm{Ob}(\mathcal{C})$. Since we also have $W \hookrightarrow k \mathcal{C} e_{m}$ and the $\mathcal{C}$-modules $k \mathcal{C} e_{1}, \ldots, k \mathcal{C} e_{r}, k \mathcal{C} e_{m}$ are injective, it follows from (7.1) by a standard argument that there exists an injective homomorphism from $F$ to $k \mathcal{C} e_{n_{1}} \oplus \cdots \oplus$ $k \mathrm{C} e_{n_{r}} \oplus k \mathrm{C} e_{m}$.

\subsection{Proof of Theorem 1.7. Suppose that $\mathcal{C}$ is $\mathrm{FI}_{G}$.}

Proof of Theorem 1.7. (i) Suppose that $V$ is a finitely generated injective $\mathcal{C}$-module. By Lemma 7.2, there is a short exact sequence $0 \rightarrow T \rightarrow V \rightarrow F \rightarrow 0$ where $T$ is a finite dimensional $\mathrm{C}$-module and $F$ is a torsion-free $\mathcal{C}$-module. For any $\mathcal{C}$-module $U$, there is a long exact sequence

$$
\cdots \rightarrow \operatorname{Hom}_{\mathcal{C}}(U, F) \rightarrow \operatorname{Ext}_{\mathcal{C}}^{1}(U, T) \rightarrow \operatorname{Ext}_{\mathcal{C}}^{1}(U, V) \rightarrow \cdots .
$$

Since $V$ is injective, one has $\operatorname{Ext}_{\mathcal{C}}^{1}(U, V)=0$. Since $F$ is torsion-free, one has $\operatorname{Ext}_{\mathcal{C}}^{1}(U, T)=0$ whenever $U$ is finite dimensional.

Choose $l \in \mathrm{Ob}(\mathcal{C})$ such that $T(m)=0$ for all $m>l$. Suppose $W$ is a finitely generated -module. By Lemma 2.4, we can choose $n>l$ such that: $\operatorname{Ext}_{\mathcal{e}}^{1}(W, T)=\operatorname{Ext}_{\mathcal{C}_{n}}^{1}\left(\jmath^{*}(W), \jmath^{*}(T)\right)$ where $\jmath: \mathcal{C}_{n} \hookrightarrow \mathcal{C}$ denotes the inclusion functor. Let $U=\jmath_{*}\left(\jmath^{*}(W)\right)$. Observe that $\jmath^{*}(U)=$ $\jmath^{*}(W)$ and $\jmath_{*}\left(\jmath^{*}(T)\right)=T$. Thus, by Lemma 2.3, one has

$$
\operatorname{Ext}_{\mathcal{C}_{n}}^{1}\left(\jmath^{*}(W), \jmath^{*}(T)\right)=\operatorname{Ext}_{\mathcal{C}}^{1}(U, T)=0 .
$$

Therefore, $\operatorname{Ext}_{\mathcal{C}}^{1}(W, T)=0$. It follows from Corollary 2.2 that $T$ is an injective $\mathcal{C}$-module.

We deduce that $V$ is isomorphic to $T \oplus F$, so $F$ is an injective $\mathcal{C}$-module. By Proposition 7.5. it follows that $F$ is a direct summand of a projective $\mathcal{C}$-module. Therefore, $F$ is a projective $\mathcal{C}$-module.

(ii) Suppose that $V$ is a finitely generated $\mathcal{C}$-module generated in degrees $\leqslant l$. We shall prove the result by induction on $l$.

We may assume, without loss of generality, that $V$ has no projective direct summands. By Lemma 7.2, there is a short exact sequence $0 \rightarrow T \rightarrow V \rightarrow F \rightarrow 0$ where $T$ is a finite dimensional $\mathcal{C}$-module and $F$ is a torsion-free $\mathcal{C}$-module. By Proposition 7.5, there is an injective homomorphism

$$
f: F \rightarrow k \mathcal{C} e_{n_{1}} \oplus \cdots \oplus k \mathcal{C} e_{n_{r}} \quad \text { for some } n_{1}, \ldots, n_{r} \in \mathrm{Ob}(\mathcal{C}) .
$$


Observe that $F$ is generated in degrees $\leqslant l$ and has no projective direct summands. Since there is no nonzero homomorphism from $F$ to $k \mathcal{C} e_{n}$ for any $n>l$, we can assume $n_{1}, \ldots, n_{r} \leqslant$ $l$. We claim that there is also no nonzero homomorphism from $F$ to $k \mathcal{C} e_{l}$. Indeed, the image of any homomorphism from $F$ to $k \mathrm{C} e_{l}$ is a submodule of $k \mathrm{C} e_{l}$ generated in degrees $\leqslant l$, but any such submodule of $k \mathrm{Ce}_{l}$ is a direct summand of $k \mathrm{Ce}_{l}$ and hence a projective $\mathcal{C}$-module. Since $F$ has no projective direct summands, it follows that any homomorphism from $F$ to $k \mathrm{C}_{l}$ must be 0 . If $l=0$, this implies that $F=0$, so $V=T$, and we are done by Lemma 2.6.

Suppose $l>0$. By the above observations, we can assume that $n_{1}, \ldots, n_{r} \leqslant l-1$. Let $W$ be the cokernel of $f$. Since $W$ is generated in degrees $\leqslant l-1$, it follows by induction hypothesis that $W$ has a finite injective resolution

$$
0 \rightarrow W \rightarrow J^{1} \rightarrow \cdots \rightarrow J^{a} \rightarrow 0
$$

in the category $\mathcal{C}$-mod. From this, we obtain a finite injective resolution

$$
0 \rightarrow F \stackrel{f}{\rightarrow} J^{0} \rightarrow J^{1} \rightarrow \cdots \rightarrow J^{a} \rightarrow 0
$$

where $J^{0}=k \mathcal{C} e_{n_{1}} \oplus \cdots \oplus k \mathcal{C} e_{n_{r}}$. Recall that by Lemma 2.6, the $\mathcal{C}$-module $T$ has a finite injective resolution in $\mathcal{C}$-mod. We conclude by the horseshoe lemma (see [17, page 37]) that $V$ has a finite injective resolution in $\mathcal{C}$-mod.

\section{Homological apPRoACH to REPRESENTATION STABILITY}

Throughout this section, we assume that $k$ is a splitting field for $G$ of characteristic 0 .

8.1. Simple modules of wreath product groups. Let $\mathcal{C}$ be $\mathrm{FI}_{G}$. Then $G_{n}=G \imath S_{n}$.

For each $m, l \in \mathrm{Ob}(\mathcal{C})$, we consider $G_{m} \times G_{l}$ as a subgroup of $G_{m+l}$ via

$$
G_{m} \times G_{l} \hookrightarrow G_{m+l}, \quad\left(\left(f_{1}, c_{1}\right),\left(f_{2}, c_{2}\right)\right) \mapsto\left(f_{1}, c_{1}\right) \odot\left(f_{2}, c_{2}\right) .
$$

If $X$ is a $k G_{m}$-module, and $Y$ is a $k G_{l}$-module, we set

$$
X \circledast Y=k G_{m+l} \otimes_{k\left(G_{m} \times G_{l}\right)}\left(X \otimes_{k} Y\right) .
$$

If $A$ is a $k G$-module, and $E$ is a $k S_{n}$-module, we write $A$ 乙 $E$ for the $k G$ 乙 $S_{n}$-module $A^{\otimes n} \otimes_{k} E$.

We denote by $\operatorname{Irr}(G)=\left\{\chi_{1}, \ldots, \chi_{r}\right\}$ the set of isomorphism classes of simple $k G$-modules; in particular, let $\chi_{1} \in \operatorname{Irr}(G)$ be the trivial class. For each $\chi \in \operatorname{Irr}(G)$, let $A(\chi)$ be a simple $k G$-module belonging to the isomorphism class $\chi \in \operatorname{Irr}(G)$. Recall that the isomorphism classes of simple $k S_{n}$-modules are parametrized by the partitions of $n$. For each partition $\lambda$ of $n$, let $E(\lambda)$ be a simple $k S_{n}$-module whose isomorphism class corresponds to $\lambda$.

If $\underline{\lambda}$ is a partition-valued function on $\operatorname{Irr}(G)$, we let

$$
\widetilde{L}(\underline{\lambda})=\left(A\left(\chi_{1}\right) \curlywedge E\left(\underline{\lambda}\left(\chi_{1}\right)\right)\right) \circledast \cdots \circledast\left(A\left(\chi_{r}\right) \curlywedge E\left(\underline{\lambda}\left(\chi_{r}\right)\right)\right) .
$$

The following result on the classification of simple $k G$ ? $S_{n}$-modules is well-known (see [11]).

Theorem 8.1. Suppose that $k$ is a splitting field for $G$ of characteristic 0 . Then the set of $\widetilde{L}(\underline{\lambda})$ for all partition-valued functions $\underline{\lambda}$ on $\operatorname{Irr}(G)$ with $|\underline{\lambda}|=n$ is a complete set of nonisomorphic simple $k G$ ? $S_{n}$-modules. 
8.2. Proof of Theorem 1.12. The homological properties of the category $\mathcal{C}$-mod proved in the previous section allow one to give a quick proof that finite generation implies condition (RS3).

For any partition-valued function $\underline{\lambda}$ on $\operatorname{Irr}(G)$, and integer $n \geqslant|\underline{\lambda}|+a$ where $a$ is the biggest part of $\underline{\lambda}\left(\chi_{1}\right)$, let

$$
L(\underline{\lambda})_{n}=\widetilde{L}(\underline{\lambda}[n]) .
$$

Proof of Theorem 1.12. As explained in subsection 1.4, it only remains to verify that condition (RS3) holds for finitely generated projective $\mathcal{C}$-modules. But by [5, Theorem 11.18], any finitely generated projective $\mathcal{C}$-module is a direct sum of $\mathcal{C}$-modules of the form $k \mathfrak{C} e_{m} \otimes_{k G_{m}}$ $L(\underline{\lambda})_{m}$ for some $m \in \mathrm{Ob}(\mathcal{C})$ and partition-valued function $\underline{\lambda}$ on $\operatorname{Irr}(G)$.

Let $n \geqslant 2 m$. Denote by $\mu$ the trivial partition of $n-m$. One has

$$
k \mathfrak{C}(m, n)=k G_{m} \circledast\left(A\left(\chi_{1}\right) \imath E(\mu)\right) .
$$

Therefore,

$$
\begin{aligned}
& k \mathfrak{C}(m, n) \otimes_{k G_{m}} L(\underline{\lambda})_{m}=L(\underline{\lambda})_{m} \circledast\left(A\left(\chi_{1}\right) \curlywedge E(\mu)\right) \\
& =\left(A\left(\chi_{1}\right) \curlywedge E\left(\underline{\lambda}[m]\left(\chi_{1}\right)\right)\right) \circledast\left(A\left(\chi_{1}\right) \curlywedge E(\mu)\right) \circledast\left(\underset{i=2}{\underset{*}{*}} A\left(\chi_{i}\right) \prec E\left(\underline{\lambda}\left(\chi_{i}\right)\right)\right) .
\end{aligned}
$$

The rest of the proof is same as [9, Lemma 2.3]. By Pieri's formula,

$$
\left(A\left(\chi_{1}\right) \prec E\left(\underline{\lambda}[m]\left(\chi_{1}\right)\right)\right) \circledast\left(A\left(\chi_{1}\right) \prec E(\mu)\right)=\bigoplus_{\nu \in P(n)} A\left(\chi_{1}\right) \prec E(\nu),
$$

where $P(n)$ denotes the set of all partitions $\nu$ whose Young diagram can be obtained from the Young diagram of $\underline{\lambda}[m]\left(\chi_{1}\right)$ by adding $n-m$ boxes with no two in the same column. Let $\hbar: P(n) \rightarrow P(n+1)$ be the map which assigns to $\nu \in P(n)$ the partition $\hbar(\nu) \in P(n+1)$ whose Young diagram is obtained from the Young diagram of $\nu$ by adding a box in the first row. It is plain that $\hbar$ is injective. Since $n \geqslant 2 m$, the map $\hbar$ must also be surjective. The result follows.

\section{REFERENCES}

[1] D. Benson, Representations and cohomology. I. Basic representation theory of finite groups and associative algebras. Second edition. Cambridge Studies in Advanced Mathematics 30. Cambridge University Press, Cambridge, 1998.

[2] T. Church, J. Ellenberg, B. Farb, FI-modules and stability for representations of symmetric groups, to appear in Duke Math J., arXiv:1204.4533.

[3] T. Church, J. Ellenberg, B. Farb, R. Nagpal, FI-modules over Noetherian rings, Geom. Top. 18-5 (2014), 2951-2984, arXiv:1210.1854

[4] T. Church, B. Farb, Representation theory and homological stability, Adv. Math. 245 (2013), 250-314, arXiv:1008.1368.

[5] T. tom Dieck, Transformation groups, de Gruyter Studies in Mathematics 8, Walter de Gruyter \& Co., Berlin, 1987.

[6] B. Farb, Representation stability, to appear in Proceedings of ICM 2014, arXiv:1404.4065

[7] W.L. Gan, L. Li, Noetherian property of infinite EI categories, arXiv:1407.8235.

[8] W.L. Gan, L. Li, Koszulity of directed categories in representation stability theory, arXiv:1411.5308. 
[9] D. Hemmer, Stable decompositions for some symmetric group characters arising in braid group cohomology, J. Combin. Theory Ser. A 118 (2011), no. 3, 1136-1139.

[10] N. Kuhn, Generic representation theory of finite fields in nondescribing characteristic, Adv. Math. 272 (2015), 598-610, arXiv:1405.0318

[11] I.G. Macdonald, Polynomial functors and wreath products, J. Pure Appl. Algebra 18 (1980), no. 2, 173204.

[12] A. Putman, S. Sam, Representation stability and finite linear groups, arXiv:1408.3694

[13] S. Sam, A. Snowden, GL-equivariant modules over polynomial rings in infinitely many variables, to appear in Trans. Amer. Math. Soc., arXiv:1206.2233

[14] S. Sam, A. Snowden, Gröbner methods for representations of combinatorial categories, arXiv:1409.1670

[15] S. Sam, A. Snowden, Representations of categories of G-maps, arXiv:1410.6054

[16] A. Snowden, Syzygies of Segre embeddings and $\Delta$-modules, Duke Math. J. 162 (2013), no. 2, 225-277, arXiv:1006.5248.

[17] C. Weibel, An introduction to homological algebra, Cambridge Studies in Advanced Mathematics 38, Cambridge University Press, Cambridge, 1994.

[18] J. Wilson, $\mathrm{FI}_{\mathcal{W}}$-modules and stability criteria for representations of classical Weyl groups, J. Algebra 420 (2014), 269-332, arXiv:1309.3817

Department of Mathematics, University of California, Riverside, CA 92521, USA

E-mail address: wlgan@math.ucr.edu

College of Mathematics and Computer Sciences, Hunan Normal University, Changsha, 410081, CHINA.

E-mail address: lipingli@hunnu.edu.cn 